\title{
Geostrophic Adjustment in an Axisymmetric Vortex
}

\author{
by \\ Wayne H. Schubert \\ James J. Hack \\ Pedro L. Silva Dias \\ Scott R. Fulton
}

Department of Atmospheric Science

Colorado State University

Fort Collins, Colorado

\section{Department of \\ Atmospheric Science}




\title{
GEOSTROPHIC ADJUSTMENT IN AN
}

AXISYMMETRIC VORTEX

\author{
by \\ Wayne H. Schubert \\ James J. Hack \\ Pedro L. Silva Dias \\ Scott R. Fulton
}

\begin{abstract}
This research was supported by the Global Atmospheric Research Program, National Science Foundation, and the GATE Project Office, NOAA under Grant No. ATM-7808125.
\end{abstract}

Department of Atmospheric Science

Colorado State University

Fort Collins, Colorado

80523

November, 1979

Atmospheric Science Paper No. 317 


\section{CONTENTS}

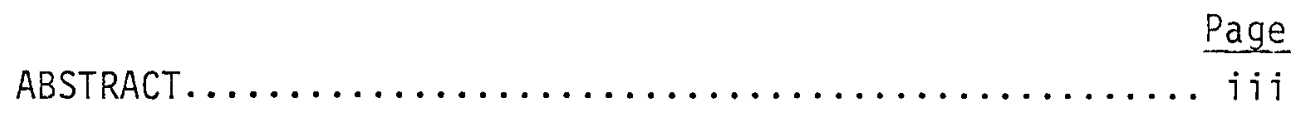

1. INTRODUCTION.........................

2. GOVERNING EQUATIONS...................... 5

3. METHOD OF SOLUTION....................... 8

3.1 Final Adjusted State..................... 8

3.2 Transient State........................ 9

4. GENERAL PROPERTIES OF THE SOLUTIONS .............. 12

5. INITIAL TOP-HAT POTENTIAL VORTICITY............. 15

5.1 Continuous Initial Tangential Wind........... 15

5.2 Discontinuous Initial Tangential Wind........... 17

5.3 Transient Solution...................... 18

6. INITIAL GAUSSIAN-TYPE POTENTIAL VORTICITY.......... 21

7. INITIAL RADIAL WIND...................... 23

8. THE FORCED CASE ......................... 25

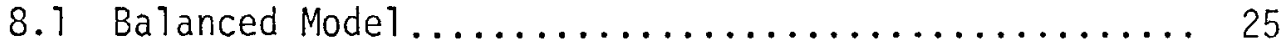

8.2 Primitive Equation Model ............... 26

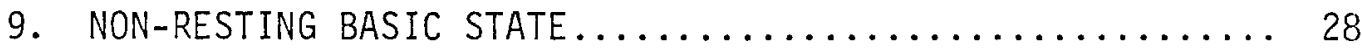

10. IMPLICATIONS FOR BOUNDARY-CONDITIONS $\ldots \ldots \ldots \ldots \ldots \ldots \ldots . \ldots \ldots$

11. CONCLUDING REMARKS ......................... 39

ACKNOWLEDGEMENTS ........................ 41

REFERENCES ........................... 42

LIST OF FIGURES ....................... 47 


\section{ABSTRACT}

A linearized system of equations for the atmosphere's first internal mode in the vertical is derived. The system governs small amplitude, forced, axisymmetric perturbations on a basic state tangential flow which is independent of height. When the basic flow is at rest, solutions for the transient and final adjusted state are found by the method of Hanke1 transforms. Two examples are considered, one with an initial top-hat potential vorticity and one with an initial Gaussian-type potential vorticity. These two examples, which extend the work of Fischer (1963) and Obukhov (1949), indicate that the energetical efficiency of cloud cluster scale heating in producing balanced vortex flow is very low, on the order of a few percent. The vast majority of the energy is simply partitioned to gravity-inertia waves. In contrast the efficiency of cloud cluster scale vorticity transport is very high.

When the basic state possesses positive relative vorticity in an. inner region, the energy partition can be substantially modified, and cloud cluster scale heating can become considerably more efficient.

The energy partition results have important implications for the lateral boundary condition used in tropical cyclone models. Faced with the fact that a perfect non-reflecting condition is possible but impractical to implement, one is forced to use an approximate condition which causes some reflection of gravity-inertia waves and hence some distortion of the geostrophic adjustment process. The distortion can be kept sma 71 by the use of a suitable radiation condition. 


\section{INTRODUCTION}

The problem of geostrophic adjustment is to determine the final adjusted state and the transient states which occur when atmospheric or oceanic flows mutually adjust the pressure field and the momentum field to a state of geostrophic balance. This problem was first studied by Rossby (1938), Cahn (1945), and Obukhov (1949). Rossby studied only the relationship between the initial unbalanced state and the final geostrophically balanced state. The linear transient adjustment was studied for the one-dimensional case by Cahn and for the two-dimensional case by Obukhov. Since these classical studies (primarily barotropic) there have been many contributions to this problem, e.g. the effect of stratification (Bolin, 1953; Kibel, 1955, 1957, 1963; Fjelsted, 1958; Monin, 1958, Fischer, 1963), the effect of horizontal shear of the basic flow (37umen and Washington, 1969), the effect of nonlinear terms (Blumen, 1967), the effect of a variable coriolis parameter (Dobrischman, 1964; Geisler and Dickinson, 1972), the effect of a transient (rather than implusive) forcing of the momentum field (Veronis, 1956) and of the mass field (Paegle, 1978). Geisler (1970) has also shown that the linear response of the ocean to a moving hurricane is similar in many respects to the problem of geostrophic adjustment. Analytic solutions to the adjustment problem also serve as useful guides in the design of finite differencing schemes for more complicated models (Arakawa and Lamb, 1977; Schoenstadt, 1977, 1979, 1980). A review of the early Soviet literature on geostrophic adjustment (and numerical weather prediction) can be found in Phillips et a1. (1960). An excellent recent and comprehensive review of the adjustment problem can be found in the paper by Blumen (1972). 
The purpose of this paper is to present some simple solutions for the geostrophic adjustment of an axisymmetric vortex and to discuss the implication of the results for the modeling of tropical cyclones. Tropical cyclone models can be divided into two classes: balanced models and primitive equation models. In balanced models (e.g. Ogura, 1964; Kuo, 1965; Ooyama, 1969a, b; Sundquist, 1970a, b) the flow is assumed to be axisymmetric and in gradient balance. Since gravity-inertia waves are then filtered, the transient aspects of the adjustment problem are not simulated. Primitive equation models may be either axisymmetric (e.g. Yamasaki, 1968a, b; Rosenthal, 1970, 1971, 1978; Kurihara, 1975) or asymmetric (e.g. Anthes et a1., 1971a, b; Anthes, 1972; Kurihara and Tuleya, 1974; Mathur, 1974; Madala and Piacsek, 1975), and in either case geostrophic adjustment becomes one of the important physical processes which must be properly simulated.

Some discussion of the geostrophic adjustment of axisymmetric flows appears in the works of Obukhov (1949), Fischer (1963), Janjic and WiinNielsen (1977) and Paegle (1978). Obukhov (1949) studies the adjustment of an initial Gaussian-type vorticity while Fischer (1963) studies the adjustment of an initial top-hat vorticity (a Rankine vortex). In section 5 and 6 we extend these results by considering both the unbalanced initial vortex problem and the unbalanced initial pressure gradient problem, and by considering the horizontal scale dependence of the energy partition between the final balanced flow and the gravity-inertia waves.

By numerical evaluation of the general integral solutions Paegle (1978) studies the adjustment of the divergent part of the wind to axisymmetric switch-on heating and diurnally oscillating heating. In 
addition he compares the non-linear gradient adjustment process with the linear geostrophic adjustment process. Our results are complementary to his but place more emphasis on simple analytical solutions.

An analysis of geostrophic adjustment in a rotating cylindrical container appears in the work of Janjic and Wiin-Nielsen (1977), who argue that motion in the atmosphere occurs in a 1 imited space, and this fact prevents an initial disturbance from traveling away to infinity. Thus, Janjic and Wiin-Nielsen study the adjustment of axisymmetric flows in a cylindrical region which has zero divergence on the outer boundary. Not surprisingly, gravity-inertia waves are reflected at the boundary and true geostrophic adjustment does not occur. Our view of the adjustment problem is considerably different. There are of course real physical situations in which gravity-inertia waves are reflected. For example, when an initial spectrum of waves propagates through a region of variable coriol is parameter, each wave will not penetrate far beyond the latitude where its frequency matches the local coriolis parameter (Geisler and Dickinson, 1972). Horizontal shear of the basic state can also alter the propagation of the gravity-inertia waves. However, these effects are considerably different than the boundary condition imposed by Janjic and Wiin-Nielsen. Our view is that, for tropical cyclone modeling, solutions for the unbounded domain are more relevant than solutions for the bounded domain. Since a circular domain of radius 1200-1300 km occupies only about $1 \%$ of the total surface area of the globe, the region surrounding a tropical cyclone can, for practical purposes, be considered infinite in extent. Then, the differences between solutions for an unbounded domain and solutions for a bounded domain should be viewed as a measure of the inadequacy of the boundary conditions which we have been forced to use in the bounded case. 
The basic outline of the present paper is as follows. After deriving in section 2 the governing equations for sma 11 amplitude perturbations (about a non-resting basic state) of the first baroclinic mode, we discuss in section 3 two riathods for the solution of these equations. The first method is based on a local conservation equation and yields the final balanced state from knowledge of the initial state, regardless of whether the basic state is at rest. The second method is based on Hankel transforms and yields the transient solution when the basic state is at rest. After discussing some general properties of the solutions (section 4), we present in sections 5-7 some simple examples of analytical solutions. Section 8 contrasts solutions of the forced balanced model with both the slowly forced and rapidly forced primitive equation model. In section 9 we illustrate how a non-resting basic state can influence the energy partition between the final balanced flow and the gravity-inertia waves. The lop-sided energy partition typical of tropical motions has important implications for the lateral boundary conditions used in tropical models. This problem is discussed in section 10 . 


\section{GOVERNING EQUATIONS}

Using cylindrical coordinates in the horizontal and using pressure as the vertical coordinate, the axisymmetric form of the primitive equations can be written

$$
\begin{gathered}
\frac{\partial u}{\partial t}+u \frac{\partial u}{\partial r}+\omega \frac{\partial u}{\partial p}-\left(f+\frac{v}{r}\right) v+\frac{\partial \phi}{\partial r}=0, \\
\frac{\partial v}{\partial t}+u \frac{\partial v}{\partial r}+\omega \frac{\partial v}{\partial p}+\left(f+\frac{v}{r}\right) u=F \alpha^{2} t e^{-\alpha t,}, \\
\frac{\partial r u}{r \partial r}+\frac{\partial \omega}{\partial p}=0, \\
\frac{\partial}{\partial t}\left(-\frac{\partial \phi}{\partial p}\right)+u \frac{\partial}{\partial r}\left(-\frac{\partial \phi}{\partial p}\right)-\sigma \omega=\frac{R Q}{c_{p} p} \alpha^{2} t e^{-\alpha t},
\end{gathered}
$$

where $u$ is the radial component of velocity, $v$ the tangential component, $\omega$ the vertical $p$ velocity $d p / d t, \phi$ the geopotential, $f$ the constant coriolis parameter, and $\sigma$ the static stability defined by $\sigma=-\frac{\alpha}{\theta} \frac{\partial \theta}{\partial p}$. Here we have included a specified source term for the rotational part of the wind field and a specified rate of external heat addition, both of which we have assumed can be factored into space dependent and time dependent parts, with the time dependent part given by $\alpha^{2}$ te ${ }^{-\alpha t}$. Sma 11 $\alpha$ corresponds to slow forcing and large $\alpha$ to rapid forcing, but the total forcing is independent of $\alpha$ since $\int_{0}^{\infty} \alpha^{2} t e^{-\alpha t} d t=1$. A source term for the divergent part of the wind has been omitted since it is not of such fundamental importance as $F$, as we shall see later. By the inclusion of $F$ and $Q$ we have in mind the apparent sources of angular momentum (or vorticity) and heat due to the effects of cumulus clouds. These effects have been extensively studied from a diagnostic point of 
view (e.g. Reed and Recker, 1971; Williams and Gray, 1973; Yanai et a1., 1973, 1976; Ogura and Cho, 1973; Reed and Johnson, 1974; Ruprecht and Gray, 1976; Chu, 1976; Hodur and Fein, 1977; Reed et a1., 1977; Shapiro, 1978; Thompson et a1., 1979; Stevens, 1979). Although the apparent sources actually depend on the flow field, we consider such complications to be beyond the scope of the present study. The specified time distribution of the forcing is arbitrary and has been simply chosen so that we can later illustrate the dependence of the adjustment process on the time scale $1 / \alpha$.

In order to simplify the vertical structure of this system as much as possible we follow the geostrophic adjustment study of Paegle (1978) by considering the standard two layer model version of (2.1)-(2.4). In addition, we restrict our attention to small perturbations about a basic state of gradient balance, i.e. $\bar{u}=\bar{\omega}=0$ and $\left(f+\frac{\bar{v}}{r}\right) \bar{v}=\frac{\partial \bar{\phi}}{\partial r}$ with $\bar{v}$ assumed to be a function of $r$ but not of $p$. With (2.1)-(2.3) applied at level $1(250 \mathrm{mb})$ and level $3(750 \mathrm{mb})$, and (2.4) applied at level $2(500 \mathrm{mb})$, and requiring that $\omega=0$ at the top and bottom pressure surfaces, (2.1)-(2.4) reduce to

$$
\begin{gathered}
\frac{\partial u_{d}}{\partial t}-\left(f+\frac{2 \bar{v}}{r}\right) v_{d}+\frac{\partial \phi_{d}}{\partial r}=0, \\
\frac{\partial v_{d}}{\partial t}+\left(f+\frac{\partial r \bar{v}}{r \partial r}\right) u_{d}=F_{d} \alpha^{2} t e^{-\alpha t}, \\
\frac{\partial \phi_{d}}{\partial t}+\frac{\bar{\sigma}_{2}(\Delta p)^{2}}{2} \frac{\partial r u_{d}}{r \partial r}=\frac{R Q_{2}}{c_{p}} \alpha^{2} t e^{-\alpha t},
\end{gathered}
$$

where $\Delta p=500 \mathrm{mb}$, and where the subscript "d" refers to the difference between the upper and lower levels, e.g. $u_{d}=u_{1}-u_{3}$. 
It is convenient to convert (2.5)-(2.7) into non-dimensional form by choosing units of time and horizontal distance as follows:

$$
\text { unit of time: } 1 / f
$$

unit of horizontal distance: $c / f$.

Here $c^{2}=\frac{\bar{\sigma}_{2}(\Delta p)^{2}}{2}$ is the square of the phase speed of pure internal gravity wave. Then the dimensionless quantities, time, radial distance, basic state tangential wind, shear velocity components, thickness, source terms and forcing time scale, become respectively $f t, \frac{f}{c} r, \frac{\vec{v}}{c}$, $\frac{u_{d}}{c}, \frac{v_{d}}{c}, \frac{\phi_{d}}{c}, \frac{F_{d}}{c}, \frac{R}{c_{p}}, \frac{Q_{2}}{c^{2}}$, and $\frac{f}{\alpha}$. Using the symbols $t, r, \bar{v}, u$, $v, \phi, F, Q$ and $1 / \alpha$ for these new dimensionless variables, (2.5)-(2.7) become

$$
\begin{aligned}
& \frac{\partial u}{\partial t}-\left(1+\frac{2 \bar{v}}{r}\right) v+\frac{\partial \phi}{\partial r}=0, \\
& \frac{\partial v}{\partial t}+(1+\bar{\zeta}) u=F \alpha^{2} t e^{-\alpha t}, \\
& \frac{\partial \phi}{\partial t}+\frac{\partial r u}{r \partial r}=Q \alpha^{2} t e^{-\alpha t},
\end{aligned}
$$

where $\bar{\zeta}$ is the basic state relative vorticity $\frac{\partial r \bar{v}}{r \partial r}$. 


\section{METHOD OF SOLUTION}

If $F=Q=0, a$ flow characterized by $u=0$ and $\left(1+\frac{2 \bar{v}}{r}\right) v=\frac{\partial \phi}{\partial r}$ is a steady state solution of (2.8)-(2.10). If the flow initially deviates from this balanced state a transient adjustment process occurs and ultimately a balanced flow results. Rossby (1938) and Obukhov (1949) first pointed out that the final balanced state can be found without solving the transient problem.

\subsection{Final adjusted state}

The final adjusted state is most easily obtained by combining (2.9) and $(2,10)$ to obtain

$$
\frac{\partial}{\partial t}\left\{\frac{\partial}{r \partial r}\left(\frac{r v}{1+\bar{\zeta}}\right)-\phi\right\}=\left\{\frac{\partial}{r \partial r}\left(\frac{r F}{1+\bar{\zeta}}\right)-Q\right\} \alpha^{2} t e^{-\alpha t},
$$

which, in the absence of forcing, is a local conservation equation. Equation (3.1) can be integrated to obtain

$$
\frac{d}{r d r}\left(\frac{r v_{\infty}}{1+\bar{\zeta}}\right)-\phi_{\infty}=\frac{d}{r d r}\left[\frac{r\left(v_{0}+F\right)}{1+\bar{\zeta}}\right]-\left(\phi_{0}+Q\right),
$$

where the subscripts $\infty$ and 0 indicate values at infinite time and initial time respectively. If we assume that the final tangential wind $v_{\infty}$ is in balance, i.e.

$$
\left(1+\frac{2 \bar{v}}{r}\right) v_{\infty}=\frac{d \phi_{\infty}}{d r}
$$

(3.2) can be written as

$$
\frac{d}{r d r}\left\{\frac{r}{\bar{s}} \frac{d \phi_{\infty}}{d r}\right\}-\phi_{\infty}=\frac{d}{r d r}\left[\frac{r\left(v_{0}+F\right)}{1+\bar{\zeta}}\right]-\left(\phi_{0}+Q\right),
$$


where $\bar{S}=\left(1+\frac{2 \bar{v}}{r}\right)(1+\bar{\zeta})$ is a measure of the inertial stability of the basic state vortex. In the case of a resting basic state (3.4) reduces to

$$
\frac{d^{2} \phi_{\infty}}{d r^{2}}+\frac{d \phi_{\infty}}{r d r}-\phi_{\infty}=\zeta_{0}-\phi_{0}+\frac{d r F}{r d r}-Q,
$$

which, in the abserice of forcing, is a statement of the local conservation of perturbation potential vorticity. Analytic solutions of (3.5) will be presented in sections 4,5 and 6 , while numerical solutions of (3.4) will be presented in section 9.

\subsection{Transient state}

Transient solutions of (2.8)-(2.10) are most easily obtained when the basic state is at rest, i.e. when $\bar{v}=\bar{\zeta}=0$. Let us suppose that $u(r, t), v(r, t), \phi(r, t), F(r)$ and $Q(r)$ are sufficiently well behaved that their Hankel transforms exist. The order $v$ Hankel transform pair is ${ }^{1}$

$$
\begin{aligned}
& \psi(r, t)=\int_{0}^{\infty} \hat{\psi}(k, t) J_{v}(k r) k d k, \\
& \hat{\psi}(k, t)=\int_{0}^{\infty} \psi(r, t) J_{v}(k r) r d r,
\end{aligned}
$$

where $J_{v}$ is the order $v$ Bessel function of the first kind. Let us define $\hat{u}(k, t), \hat{v}(k, t)$ and $\hat{F}(k)$ as the first order Hankel transforms of $u(r, t), v(r, t)$ and $F(r)$; in addition let us define $\hat{\phi}(k, t)$ and $\hat{Q}(k)$ as the zero order transforms of $\phi(r, t)$ and $Q(r)$.

Other possibilities for the definition of the Hankel transform pair exist. For example, that used by Erdelyi et al. (1954) is written in such a way that it reduces to a Fourier transform when $v= \pm \frac{1}{2}$. We have chosen the form given by (3.6) and (3.7) since it makes the following analysis somewhat simpler. 
Assuming a basic state of rest, we can take the first order transforms of (2.8) and (2.9) and the zero order transform of (2.10) to obtain

$$
\begin{aligned}
& \frac{d \hat{u}}{d t}-\hat{v}-k \hat{\phi}=0, \\
& \frac{d \hat{v}}{d t}+\hat{u}=\hat{F} \alpha^{2} t e^{-\alpha t}, \\
& \frac{d \hat{\phi}}{d t}+\hat{k} \hat{u}=\hat{Q} \alpha^{2} t e^{-\alpha t} .
\end{aligned}
$$

If we define $\hat{u}_{0}(k)$ and $\hat{v}_{0}(k)$ as the first order transforms of the initial winds $u(r, 0)$ and $v(r, 0)$, and $\hat{\phi}_{0}(k)$ as the zero order transform of the initial geopotential $\phi(r, 0)$, we can write the solutions of $(3.8)-(3.10)$ as

$$
\begin{aligned}
& \hat{u}(k, t)=\left\{\hat{u}_{0}-\frac{2 \alpha^{3}}{\left(\alpha^{2}+v^{2}\right)^{2}}(\hat{F}+k \hat{Q})\right\} \cos v t \\
&+\left\{\hat{v}_{0}+k \hat{\phi}_{0}+\frac{\alpha^{2}\left(\alpha^{2}-v^{2}\right)}{\left(\alpha^{2}+v^{2}\right)^{2}}(\hat{F}+k \hat{Q})\right\} v^{-1} \sin v t \\
&+\frac{\alpha^{3}}{v^{2}\left(\alpha^{2}+v^{2}\right)}(\hat{F}+k \hat{Q})\left(\frac{2 v^{2}}{\alpha^{2}+v^{2}}-\alpha t\right) e^{-\alpha t} \\
&+(\hat{F}+k \hat{Q}) v^{-2} \alpha^{2} t e^{-\alpha t}, \\
& \hat{v}(k, t)=\left\{\hat{v}_{0}+k \hat{\phi}_{0}+\frac{\alpha^{2}\left(\alpha^{2}-v^{2}\right)}{\left(\alpha^{2}+v^{2}\right)^{2}}(\hat{F}+k \hat{Q})\right\} v^{-2} \cos v t \\
& \\
&-\left\{\hat{u}_{0}-\frac{2 \alpha^{3}}{\left(\alpha^{2}+v^{2}\right)^{2}}(\hat{F}+k \hat{Q})\right\} v^{-1} \sin v t \\
&-\frac{\alpha^{2}}{v^{2}\left(\alpha^{2}+v^{2}\right)}(\hat{F}+k \hat{Q})\left(\frac{\alpha^{2}-v^{2}}{\alpha^{2}+v^{2}}+\alpha t\right) e^{-\alpha t} \cdot \\
&+\left\{k \hat{v}_{0}-\hat{\phi}_{0}+(k \hat{F}-\hat{Q})\left[1-(1+\alpha t) e^{-\alpha t}\right]\right\} k v^{-2},
\end{aligned}
$$




$$
\begin{aligned}
\hat{\phi}(k, t)= & \left\{\hat{v}_{0}+k \hat{\phi}_{0}+\frac{\alpha^{2}\left(\alpha^{2}-v^{2}\right)}{\left(\alpha^{2}+v^{2}\right)^{2}}(\hat{F}+k \hat{Q})\right\} k v^{-2} \cos v t \\
& -\left\{\hat{u}_{0}-\frac{2 \alpha^{3}}{\left(\alpha^{2}+v^{2}\right)^{2}}(\hat{F}+k \hat{Q})\right\} k v^{-1} \sin v t \\
& -\frac{\alpha^{2} k}{v^{2}\left(\alpha^{2}+v^{2}\right)}(\hat{F}+k \hat{Q})\left(\frac{\alpha^{2}-v^{2}}{\alpha^{2}+v^{2}}+\alpha t\right) e^{-\alpha t} \\
& -\left\{k \hat{v}_{0}-\hat{\phi}_{0}+(k \hat{F}-\hat{Q})\left[1-(1+\alpha t) e^{-\alpha t}\right]\right\} v^{-2},
\end{aligned}
$$

where $\nu=\left(1+k^{2}\right)^{\frac{1}{2}}$. In the absence of forcing these solutions are the axisymmetric two-dimensional analogues of the one-dimensional case discussed by Schoenstadt $(1977,1979)$. A11 the terms on the right hand sides of (3.11)-(3.13) are associated with the transient solution except the last term in (3.12) and the last term in (3.13), which are associated with the geostrophically balanced state. From (3.12) and (3.13) it can be seen that the potential vorticity $(k \hat{v}-\hat{\phi})$ associated with the transient part of the solution is zero while that associated with the balanced part is $k \hat{v}_{0}-\hat{\phi}_{0}+(k \hat{F}-\hat{Q})\left[1-(1+\alpha t) e^{-\alpha t}\right]$, i.e. the initial potential vorticity plus the forced potential vorticity. 


\section{GENERAL PROPERTIES OF THE SOLUTIONS}

Before proceeding to specific examples let us consider some of the . general properties of the solutions (3.11)-(3.13). The first two terms in the right hand side of (3.11)-(3.13) are terms which are oscillatory in time in spectral space. In physical space they represent propagating gravity-inertia waves. If one waits long enough for the forcing to be essentially completed and for the gravity-inertia waves to disperse to a great distance, only the final balanced flow remains. Then, (3.12) and (3.13) yieid

$$
\begin{aligned}
& \hat{v}_{\infty}=\frac{k^{2}}{1+k^{2}}\left(\hat{v}_{0}+\hat{F}\right)+\frac{1}{1+k^{2}}\left(\hat{v}_{g o}+\hat{F}_{g}\right), \\
& \hat{\phi}_{\infty}=\frac{k^{2}}{1+k^{2}}\left(\hat{\phi}_{g o}+\hat{Q}_{g}\right)+\frac{1}{1+k^{2}}\left(\hat{\phi}_{0}+\hat{Q}\right),
\end{aligned}
$$

where $\hat{v}_{\text {go }}, \hat{F}_{g}, \hat{\phi}_{\text {go }}$ and $\hat{Q}_{g}$ are defined by

$$
\begin{array}{ll}
\hat{v}_{\text {go }}=-k \hat{\phi}_{0}, & \hat{F}_{g}=-k \hat{Q}, \\
\hat{v}_{0}=-k \hat{\phi}_{g 0}, & \hat{F}=-k \hat{Q}_{g} .
\end{array}
$$

Equation (4.1) states that the final wind $\hat{v}_{\infty}$ is a weighted average of the initial wind $\hat{v}_{0}$ and forced wind $\hat{F}$ (weight $\frac{k^{2}}{1+k^{2}}$ ) and the initial geostrophic wind $\hat{v}_{\text {go }}$ and forced geostrophic wind $\hat{F}_{g}$ (weight $\frac{1}{1+k^{2}}$ ). A similar interpretation holds for (4.2). The two important weighting functions are shown in Fig. 1. We may interpret $\frac{k^{2}}{1+k^{2}}$ as the spectral modification of a tangential wind forcing or an initial tangential wind disturbance and note that low wave numbers are eliminated. Likewise, $\frac{1}{1+k^{2}}$ is the spectral modification of a heat source or an initial pressure disturbance, with high wave numbers being eliminated. 
Because of the equivalence of $\hat{v}_{0}$ and $\hat{F}$ and of $\hat{\phi}_{0}$ and $\hat{Q}$ as far as the final adjusted state is concerned, let us for the moment consider the unforced case. To see the energy implications of (4.1) and (4.2) we note that, from the Parceval relation, the kinetic energy associated with the tangential component and the available potential energy can be written

$$
\begin{aligned}
& K=\int_{0}^{\infty} \frac{1}{2} v^{2} r d r=\int_{0}^{\infty} \frac{1}{2} \hat{v}^{2} k d k, \\
& P=\int_{0}^{\infty} \frac{1}{2} \phi^{2} r d r=\int_{0}^{\infty} \frac{1}{2} \hat{\phi}^{2} k d k .
\end{aligned}
$$

If we assume that there is no radial flow initially all the kinetic energy of the initial and final states is associated with $v$. Then (4.1) and (4.2), together with (4.4) and (4.5), imply that if $\hat{\phi}_{0}=0$ (no initial pressure disturbance)

$$
\frac{k_{\infty}+P_{\infty}}{k_{0}}=\frac{\int_{0}^{\infty} \frac{k^{2}}{1+k^{2}} \hat{v}_{0}^{2} k d k}{\int_{0}^{\infty} \hat{v}_{0}^{2} k d k},
$$

while if $\hat{v}_{0}=0$ (no initial tangential wind)

$$
\frac{k_{\infty}+P_{\infty}}{P_{0}}=\frac{\int_{0}^{\infty} \frac{1}{1+k^{2}} \hat{\phi}_{0}^{2} k d k}{\int_{0}^{\infty} \hat{\phi}_{0}^{2} k d k} .
$$

The ratios $\frac{K_{\infty}+P_{\infty}}{K_{0}}$, and $\frac{K_{\infty}+P_{\infty}}{P_{0}}$ represent the fractions of the initial energies which end up in balanced flow. The remaining fractions are partitioned to gravity-inertia waves. 
Equations (4.6) and (4.7) imply the following two important rules for tropical disturbances, which usually have a horizonal scale small compared to the deformation radius.

(i) For small scale initial disturbances in the tangential wind field most of the initial energy ends up in the geostrophic flow. Thus, for smalt scale momentum forcing the efficiency of geostrophic energy generation is very high.

(ii) For small scale initial disturbances in the pressure field most of the initial energy ends up in gravity-inertia waves. Thus, for small scale heating the efficiency of geostrophic energy generation is very low.

In order to see just how low or high these efficiencies can be for tropical weather systems we shall present examples in sections 5 and 6 . 
5. INITIAL TOP-HAT POTENTIAL VORTICITY

5.1 Continuous initial tangential wind

Let us assume that the initial potential vorticity is given by

$$
\zeta_{0}-\phi_{0}= \begin{cases}\frac{2}{a^{2}} & r<a \\ 0 & r>a\end{cases}
$$

One interpretation of (5.1) is that

$$
\zeta_{0}=0 \text { and } \phi_{0}=\left\{\begin{array}{cc}
-\frac{2}{a^{2}} & r<a \\
0 & r>a,
\end{array}\right.
$$

i.e. there is no initial vorticity but the amount of "mass" $2 \pi$ has been removed from the region $r<a$. A second possible interpretation is that there is ro initial geopotential perturbation but the initial tangential wind has the form of a Rankine vortex, i.e.

$$
\phi_{0}=0 \text { and } r v_{0}=\left\{\begin{array}{cc}
\frac{r^{2}}{a^{2}} & r<a \\
1 & , r \geq a .
\end{array}\right. \text {. }
$$

With this initial condition the initial vorticity becomes quite concentrated as the radius of maximum wind decreases but the circulation around any radius $r \geq a$ is $2 \pi$. Note also that $v_{0}$ is continuous at $r=a$.

The solution of (3.5) and (5.1) which remains bounded at the origin and at infinity and which possesses continuous $\phi_{\infty}$ and $v_{\infty}$ at $r=a$ is

$$
\phi_{\infty}=\left\{\begin{array}{ll}
-\frac{2}{a}\left[\frac{1}{a}-K_{7}(a) I_{0}(r)\right] & r \leq a \\
-\frac{2}{a} I_{7}(a) K_{0}(r) & r>a
\end{array},\right.
$$




$$
v_{\infty}=\frac{d \phi_{\infty}}{d r}=\left\{\begin{array}{lll}
\frac{2}{a} & K_{7}(a) I_{1}(r) & r \leq a \\
\frac{2}{a} I_{1}(a) K_{7}(r) & r>a
\end{array},\right.
$$

where $I_{v}$ and $k_{v}$ are the order $v$ modified Bessel functions. The final vorticity is easily computed from (5.5) or from (5.4) and the invariance of $\zeta-\phi$. The result is

$$
\zeta_{\infty}=\frac{d\left(r v_{\infty}\right)}{r d r}=\left\{\begin{array}{ccc}
\frac{2}{a} & K_{1}(a) I_{0}(r) & r<a \\
-\frac{2}{a} & I_{1}(a) K_{0}(r) & r>a
\end{array} .\right.
$$

The final geopotential $\phi_{\infty}$, tangential wind $v_{\infty}$ and vorticity $\zeta_{\infty}$ for different values of a are shown in figures 2-4. From Fig. 2 and the interpretation (5.2) we see that for a large scale pressure disturbance ( $a>1$ ) there is little change in the pressure field. From Fig. 3 and the interpretation (5.3) we see that for a radius of maximum wind smal1 compared to the radius of deformation $(a<1)$, there is little change in the tangential wind and vorticity fields.

A simple way of computing the total energy of the final adjusted state is to note that in this special case

$$
K_{\infty}+P_{\infty}=\frac{1}{2} \int_{0}^{a-} \phi_{\infty}\left(\phi_{0}-\zeta_{0}\right) r d r+\frac{a}{2} \phi_{\infty}(a)\left[v_{\infty}(a-)-v_{\infty}(a+)\right] .
$$

Since the tangential wind remains continuous the last term in (5.7) vanishes. Since the initial available potential energy associated with (5.2) is $P_{0}=1 / a^{2}$, we can derive from (5.4) and (5.7)

$$
\frac{K_{\infty}+P_{\infty}}{P_{0}}=1-2 K_{7}(a) I_{7}(a) \text {. }
$$


The separation of the final total energy $K_{\infty}+P_{\infty}$ into $K_{\infty}$ and $P_{\infty}$ can be accomplished by using (4.4) and (4.5) with $\phi$ and $v$ replaced by $\phi_{\infty}$ and $v_{\infty}$, which are given by (5.4) and (5.5). The integration of the square of the modif:ed Bessel functions can be carried out by the use of $r I_{0}^{2}(r)=\frac{d}{d r}\left\{\frac{r^{2}}{2}\left[I_{0}^{2}(r)-I_{1}^{2}(r)\right]\right\}, r I_{1}^{2}(r)=\frac{d}{d r}\left\{\frac{r^{2}}{2}\left[I_{1}^{2}(r)-I_{0}(r) I_{2}(r)\right]\right\}$, and the analogous relations for $r k_{0}^{2}(r)$ and $r k_{1}^{2}(r)$. The results are

$$
\begin{gathered}
\frac{K_{\infty}}{P_{0}}=-1+2 I_{1}(a)\left[a K_{2}(a)-K_{1}(a)\right], \\
\frac{P_{\infty}}{P_{0}}=2-2 I_{1}(a) a K_{2}(a) .
\end{gathered}
$$

The energy relations (5.8)-(5.10) are plotted in Fig. 5a. For a tropical cloud cluster of radius $300 \mathrm{~km}$ in a region with a deformation radius of $1500 \mathrm{~km}$, the dimensionless scale a is 0.2 . Fig. 5a then shows that only about $4 \%$ of the initial energy remains in the final balanced flow while about $96 \%$ is partitioned to the outward propagating gravity-inertia waves.

A similar discussion of the energetics of the initial Rankine vortex is not possible because the initial kinetic energy associated with (5.3) is not finite.

\subsection{Discontinuous initial tangential wind}

Because of the difficulty in determining the energetics of (5.3) let us consider the initial condition

$$
\phi_{0}=0 \text { and } r v_{0}= \begin{cases}\frac{r^{2}}{a^{2}} & r<a \\ 0 & r>a .\end{cases}
$$


The solution of (3.5) and (5.11) is similar to that for the initial condition (5.3) except that $v_{\infty}$ should possess the same discontinuity as $v_{0}$ at $r=a$. The solution is

$$
\begin{aligned}
& \phi_{\infty}=\left\{\begin{array}{cc}
K_{2}(a) I_{0}(r)-\frac{2}{a^{2}} & r<a \\
I_{2}(a) K_{0}(r) & r>a,
\end{array},\right. \\
& v_{\infty}= \begin{cases}K_{2}(a) I_{1}(r) & r<a \\
I_{2}(a) K_{1}(r) & r>a,\end{cases} \\
& \zeta_{\infty}= \begin{cases}K_{2}(a) I_{0}(r) & r<a \\
I_{2}(a) K_{0}(r) & r>a .\end{cases}
\end{aligned}
$$

Noting that (5.11) implies that the initial kinetic energy $k_{0}=1 / 8$, we can use (5.7) to obtain

$$
\frac{K_{\infty}+P_{\infty}}{K_{0}}=4 K_{2}(a) I_{2}(a) \text {. }
$$

Following the same procedure used in deriving (5.9) and (5.10) we can also obtain

$$
\begin{aligned}
& \frac{K_{\infty}}{K_{0}}=-2+4 K_{2}(a)\left[a I_{1}(a)-I_{2}(a)\right], \\
& \frac{P_{\infty}}{K_{0}}=2-4 K_{2}(a)\left[a I_{1}(a)-2 I_{2}(a)\right],
\end{aligned}
$$

The energy relations (5.15)-(5.17) are plotted in Fig. 5b. In general the initial wind case shows just the opposite energetic characteristics as the initial geopotential case. For example, in the initial wind 
case for $a=0.2$, about $99 \%$ of the initial energy remains in the final balanced flow while about $1 \%$ is partitioned to gravity inertia waves.

\subsection{Transient solution}

As an example of the transient adjustment let us consider the case where $F=Q=u_{0}=\phi_{0}=0$ and $v_{0}$ is the Rankine vortex given by (5.3). Equation (3.11) then reduces to

$$
\hat{u}(k, t)=\hat{v}_{0}(k) \nu^{-1} \sin v t \text {, }
$$

where ${ }^{1}$

$$
k \hat{v}_{0}(k)=k \int_{0}^{\infty} v_{0}(r) J_{1}(k r) r d r=2(k a)^{-1} J_{1}(k a) .
$$

The inverse Hankel transform (3.6) then gives

$$
u(r, t)=\int_{0}^{\infty}\left(1+k^{2}\right)^{-\frac{1}{2}} 2(k a)^{-1} J_{1}(k a) \sin \left[\left(1+k^{2}\right)^{\frac{1}{2}} t\right] J_{1}(k r) d k .
$$

If we let all the initial vorticity become concentrated at the origin $(a \rightarrow 0)$, then $(k a)^{-1} J_{1}(k a) \rightarrow \frac{1}{2}$, in which case $(5.20)$ can be evaluated to obtain

$$
r u(r, t)=\left\{\begin{array}{cl}
\sin t-\sin \left[\left(t^{2}-r^{2}\right)^{\frac{1}{2}}\right] & t>r \\
\sin t & t<r .
\end{array}\right.
$$

The divergence computed from (5.21) is given by

$$
\frac{\partial(r u(r, t))}{r \partial r}=\left\{\begin{array}{cl}
\left(t^{2}-r^{2}\right)^{-\frac{1}{2}} \cos \left[\left(t^{2}-r^{2}\right)^{\frac{1}{2}}\right] & t>r \\
0 & t<r .
\end{array}\right.
$$

The Hankel transforms used here and in the following sections can be found in Erdelyi et al. (1954), Volume II.

${ }^{2}$ A solution similar to (5.21) appears in the work of Fischer (1963). In Fischer's paper, if $r$ on the right hand side of (5.21) is replaced by a, the resulting function gives the time dependence of the divergence at the origin for an initial Rankine vortex of arbitrary radius a. In contrast our result holds at any radius $r$ but only for an initial point vortex $(a \rightarrow 0)$. 
Isolines of the radial mass flux $r u(r, t)$ in the $(r, t)$ plane are shown in Fig. 6. Plots of the divergence as a function of time for $r=\pi / 4$ and $r=3 \pi / 4$ are shown in Fig. 7. The geopotential field, computed from the time integration of (5.22), is shown in Fig. 8. For $t \gg r(5.21)$ and (5.22) become $u \sim \frac{r}{2 t} \cos t$ and $\frac{\partial r u}{r \partial r} \sim \frac{\cos t}{t}$, which show the $t^{-1}$ decay characteristic of adjustment in two dimensions (Obukhov, 1949).

The transient solution can be summarized as follows. For $t<r$ fluid particles are undergoing a pure inertial oscillation, with zero associated divergence. At $t=r$ the influence of the initial vorticity at the origin is first felt. There is then a spike in the divergence field, followed by oscillation and gradual decay as $t \rightarrow \infty$. Most of the lowering of the geopotential is associated with the spike in the divergence. Fig. 7 shows that this spike becomes narrower as $r$ increases.

In order to check the consistency of the transient results with the final adjusted state obtained in section 5.1 we note that the net effect of the oscillatory divergence field on the geopotential field is given by the integrated divergence, i.e.

$$
\phi_{\infty}=-\int_{r}^{\infty} \frac{\cos \left[\left(t^{2}-r^{2}\right)^{\frac{1}{2}}\right]}{\left(t^{2}-r^{2}\right)^{\frac{1}{2}}} d t .
$$

A change of variable allows (5.23) to be written

$$
\phi_{\infty}=-\int_{0}^{\infty} \cos (r \sinh \tau) d \tau,
$$

which is the integral representation of the zero order modified Bessel function. Thus, $\phi_{\infty}=-K_{0}(r)$ and $v_{\infty}=K_{f}(r)$, which are consistent with (5.4) and (5.5). 


\section{INITIAL GAUSSIAN-TYPE POTENTIAL VORTICITY}

In this section we consider a second simple example, one in which the initial potential vorticity is given by

$$
\zeta_{0}-\phi_{0}=\frac{2}{a^{2}}\left(\frac{4+a^{2}}{3+a^{2}}\right)\left\{1-\frac{r^{2}}{2 a^{2}}\left[1+\frac{4-\frac{r^{2}}{a^{2}}}{4+a^{2}}\right]\right\} e^{\frac{1}{2}\left(1-\frac{r^{2}}{a^{2}}\right)} .
$$

One interpretation is that $\zeta_{0}=0$ and $-\phi_{0}$ is given by the right hand side of (6.7). A second interpretation is that $\phi_{0}=0$ and $\zeta_{0}$ is given by the right hand side of (6.7), which implies that

$$
v_{0}=\left(\frac{4+a^{2}-\frac{r^{2}}{a^{2}}}{3+a^{2}}\right) \frac{r}{a^{2}} e^{\frac{3}{2}\left(1-\frac{r^{2}}{a^{2}}\right)},
$$

a vortex with circulation $2 \pi$ at $r=a$. The vortex (6.2) was first studied by Obukhov (1949).

The solution of (3.5) and (6.1) which remains bounded at the origin and at infinity is

$$
\begin{aligned}
& \phi_{\infty}=-\left(\frac{2-\frac{r^{2}}{a^{2}}}{3+a^{2}}\right) e^{\frac{1}{2}\left(1-\frac{r^{2}}{a^{2}}\right)}, \\
& v_{\infty}=\left(\frac{4-\frac{r^{2}}{a^{2}}}{3+a^{2}}\right) \frac{r}{a^{2}} e^{\frac{1}{2}\left(1-\frac{r^{2}}{a^{2}}\right)} .
\end{aligned}
$$

For the $\zeta_{0}=0$ interpretation of (6.1) the comparison of $\phi_{0}$ and $\phi_{\infty}$ for $a=5$ and $a=\frac{1}{5}$ is made in Fig. 9, which shows that for large $a, \phi_{0}$ and $\phi_{\infty}$ are nearly identical. For the $\phi_{0}=0$ interpretation the comparison of $v_{0}$ and $v_{\infty}$ for the same two a's is shown in Fig. 10. When a is small 
$v_{0}$ and $v_{\infty}$ are nearly the same, a fact which is also easily seen by comparing (6.2) and (6.4).

For the case when $\zeta_{0}=v_{0}=k_{0}=0$ we may substitute the right hand side of (6.1) into (4.5) and evaluate the integral to obtain $P_{0} \cdot K_{\infty}$ and $P_{\infty}$ may be similarly obtained by substituting (6.4) and (6.3) into (4.4) and (4.5). This results in

$$
\begin{aligned}
& \frac{K_{\infty}}{P_{0}}=\frac{3 a^{2}}{a^{4}+6 a^{2}+12}, \\
& \frac{P_{\infty}}{P_{0}}=\frac{a^{4}}{a^{4}+6 a^{2}+12},
\end{aligned}
$$

which are shown in Fig. 11a. For the case when $\phi_{0}=P_{0}=0$ a similar procedure yields

$$
\begin{aligned}
& \frac{K_{\infty}}{K_{0}}=\frac{6}{a^{4}+4 a^{2}+6}, \\
& \frac{P_{\infty}}{K_{0}}=\frac{2 a^{2}}{a^{4}+4 a^{2}+6},
\end{aligned}
$$

which are shown in Fig. 11b. The total energy curves in Figs. 11a and 11b show the same characteristics as the example given in Fig. 5. An interesting feature of Fig. 1 lb for large a is that an equipartition of energy between $K_{\infty}$ and $P_{\infty}$ does not occur. Such an equipartition does occur in the example shown in Fig. 5 b. 


\section{INITIAL RADIAL WIND}

In (2.1) we omitted the source term for the divergent part of the wind. Such a source term or a nonvanishing initial condition on $u$ is not of such fundamental importance as $F, Q, V_{0}$ and $\phi_{0}$. The reason for this is that the local conservation equations (3.4) and (3.5) will not contain such effects, i.e. the final adjusted state is independent of

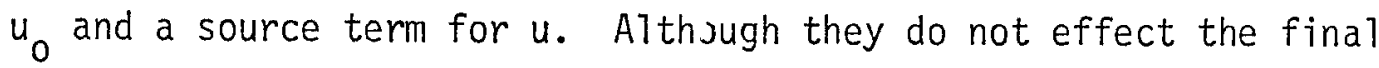
adjusted state, they can have interesting transient effects on the vorticity and geopotential fields. We illustrate these effects by considering a simple example with $F=Q=v_{0}=\phi_{0}=0$ and $r u_{0}=1-e^{-r}$, i.e. at the origin the radial mass flux vanishes but as $r$ increases the radial mass flux increases until it attains the value of unity, resulting in an initial divergence which is concentrated near the origin. Equation (3.11) then reduces to

$$
\hat{u}(k, t)=\hat{u}_{0}(k) \cos v t,
$$

where

$$
\hat{u}_{0}(k)=\int_{0}^{\infty}\left(1-e^{-r}\right) J_{1}(k r) d r=k^{-1}\left(1+k^{2}\right)^{-\frac{1}{2}} .
$$

The inverse Hankel transform (3.6) then gives

$$
u(r, t)=\int_{0}^{\infty} \frac{\cos \left[\left(1+k^{2}\right)^{\frac{1}{2}} t\right]}{\left(1+k^{2}\right)^{\frac{1}{2}}} J_{j}(k r) d k,
$$

which can also be written

$$
r u(r, t)= \begin{cases}\cos t-\cos \left[\left(t^{2}-r^{2}\right)^{\frac{1}{2}}\right] & t>r \\ \cos t-e^{-\left(r^{2}-t^{2}\right)^{\frac{1}{2}}} & t<r .\end{cases}
$$


The divergence computed from (7.4) is

$$
\frac{\partial(r u(r, t))}{r \partial r}= \begin{cases}-\left(t^{2}-r^{2}\right)^{-\frac{1}{2}} \sin \left[\left(t^{2}-r^{2}\right)^{\frac{1}{2}}\right] & t>r \\ \left(r^{2}-t^{2}\right)^{-\frac{1}{2}} e^{-\left(r^{2}-t^{2}\right)^{\frac{1}{2}}} & t<r .\end{cases}
$$

Isolines of the radial mass flux $r u(r, t)$ in the $(r, t)$ plane are shown in Fig. 12. Plots of the divergence as a function of time for $r=\pi / 4$ and $r=3 \pi / 4$ are shown in Fig. 13. The geopotential field, computed from the time integration of (7.5), is shown in Fig. 14 .

The time integrated divergence does not have a net effect on the geopotential field, which eventually returns to its original flat shape. Since potential vorticity conservation requires that the vorticity is always equal to the geopotential in this case, Fig. 14 can aiso be interpreted as the vorticity. 


\section{THE FORCED CASE}

In this section we shall illustrate how the adjustment process depends on the time scale of the forcing. To do this let us consider the case where the basic state is at rest and $u_{0}=v_{0}=\phi_{0}=F=0$.

\subsection{Balanced Mode 1}

For purposes of comparison we now derive solutions of the balanced mode 1 version of $(2.8)-(2.10)$, i.e.

$$
\begin{gathered}
v=\frac{\partial \phi}{\partial r}, \\
\frac{\partial v}{\partial t}+u=0, \\
\frac{\partial \phi}{\partial t}+\frac{\partial r u}{r \partial r}=Q \alpha^{2} t e^{-\alpha t} .
\end{gathered}
$$

If we assume that $Q$ vanishes outside $r=a$ and is constant $\left(\frac{2}{a^{2}}\right)$ inside $r=a$, and if we eliminate $u$ and $v$ from (8.1)-(8.3), we obtain

$$
\frac{\partial}{\partial t}\left\{\frac{\partial}{r \partial r}\left(r \frac{\partial \phi}{\partial r}\right)-\phi\right\}= \begin{cases}\frac{2}{a^{2}} \alpha^{2} t e^{-\alpha t} & r<a \\ 0 & r>a .\end{cases}
$$

The solution of ( 8.4$)$ is analogous to (5.4) and can be written

$$
\phi(r, t)= \begin{cases}-\frac{2}{a}\left[\frac{1}{a}-K_{1}(a) I_{0}(r)\right]\left[1-(1+\alpha t) e^{-\alpha t}\right] & r<a \\ -\frac{2}{a} I_{1}(a) k_{0}(r)\left[1-(1+\alpha t) e^{-\alpha t}\right] & r>a .\end{cases}
$$

The solution for $u$ and $v$ can be easily obtained using (8.1) and (8.2). The balanced solution (8.5) for $a=1 / 5$ is depicted in Fig. 15a. 


\subsection{Primitive Equation Model}

The solution of the primitive equation model for this case is obtained from (3.6) and (3.13), which, together with $\hat{Q}=2(k a)^{-1} J_{1}(k a)$, yield

$$
\begin{gathered}
\phi(r, t)=\int_{0}^{\infty} \hat{\phi}(k, t) J_{0}(k r) k d k \\
\hat{\phi}(k, t)= \\
\left(\alpha^{2}+v^{2}\right)^{-2}\left\{\alpha^{2}\left(\alpha^{2}-v^{2}\right) k^{2} \cos v t+2 \alpha^{2} v k^{2} \sin v t-\alpha^{2} k^{2}\left(\frac{\alpha^{2}-v^{2}}{\alpha^{2}+v^{2}}+\alpha t\right) e^{-\alpha t}\right. \\
\left.+\left[1-(1+\alpha t) e^{-\alpha t}\right]\right\} v^{-2} 2(k a)^{-1} J_{1}(k a)
\end{gathered}
$$

The balanced solution given by ( 8.5$)$ is also obtained from the inversion of the last term in (8.7). The remaining terms are associated with the gravity-inertia wave field. An asymptotic approximation to the gravityinertia wave terms can be obtained by using the method of stationary phase for Hankel transform integrals (Miles, 1971, Chapter 4). Here we present exact results, which can be obtained by the numerical evaluation of (8.6) and (8.7).

Figs. 15b. and $15 \mathrm{c}$ show $\phi(r, t)$ for $\alpha=\frac{1}{2}$ and $\alpha=2$ respectively. In both cases $a=1 / 5$. Figs. 15 a through $15 \mathrm{c}$ have been constructed with a time axis of at. When at $=5$ ninety-six percent of the eventual total forcing has already occurred. Although the final state will be the same in the three cases shown in Fig. 15 (e.g. see Eq. (3.5)), the transient states are different. In the case of rapid forcing $(\alpha=2)$ a large gravity-inertia wave front propagates outward and large time oscillations occur. This is in contrast to the slow forcing $\left(\alpha=\frac{1}{2}\right)$ case, where only a small amount of gravity-inertia wave activity is excited. The balanced model result can be considered a slightly smoothed version of the slow 
forcing case. The similarity of Figs. $15 \mathrm{a}$ and $15 \mathrm{~b}$ is, of course, support for the use of balanced models when the time scale of the forcing is large compared to $1 / f$. A criticism of the use of balanced models for tropical studies is that the vertical motion patterns associated with gravity-inertia waves can interact noninearly with the moisture field, a process which cannot be simulated with a filtered model. 


\section{NON-RESTING BASIC STATE}

In order to understand the effects of a basic state tangential flow on the adjustment nrocess we derived in section 3 the $10 \mathrm{ca} 1$ conservation relation (3.4), which is the analogue in circular geometry of the equation solved by BTumen and Washington (1969) in their study of the effects of horizontal shear on geostrophic adjustment in a barotropic fluid.

Equation (3.4) can be regarded as the differential equation for $\phi_{\infty}$ when the basic state, the initial conditions and the forcing are all known. We might consider the problem where $v_{0}=\phi_{0}=0$ and $F \neq 0, Q \neq 0$, or alternatively the problem where $F=Q=0$ and $v_{0} \neq 0, \phi_{0} \neq 0$. If these two problems result in the same radial distribution for the right hand side of (3.4), they are equivalent in the sense that they produce the same final adjusted state. As we saw in section 8 , they do not necessarily produce the same transient response since slow forcing tends to result in smooth transient solutions which are never far from gradient balance while impulsive forcing tends to result in highly oscillatory transient solutions. For simplicity we limit our discussion to the final adjusted state and to the case where $F=Q=0$.

In order to solve (3.4) we specify the inner boundary condition to be

$$
\frac{d \phi_{\infty}}{d r}=0 \text { at } r=0
$$

To derive an outer boundary condition we note that if $R$ denotes a radius large enough such that $1+\bar{\zeta}$ and $\bar{S}$ approach unity and the right hand side (3.4) approaches zero, then (3.4) becomes 


$$
\frac{d^{2} \phi_{\infty}}{d r^{2}}+\frac{d \phi_{\infty}}{r d r}-\phi_{\infty}=0 \text { for } r \geq R
$$

which is the order zero modified Bessel equation. The solution which is bounded for $r \rightarrow \infty$ behaves as $\phi_{\infty} \sim$ constant $\cdot K_{0}(r)$. Since $\frac{d K_{0}(r)}{d r}=-K_{j}(r)$, our boundary condition is

$$
K_{0}(r) \frac{d \phi_{\infty}}{d r}+K_{7}(r) \phi_{\infty}=0 \text { at } r=R
$$

Using the boundary conditions (9.1) and (9.3) we can compute solutions of (3.4) using the numerical procedure given by Richtmyer and Morton (1967). Once $\phi_{\infty}$ is determined, $v_{\infty}$ can be computed from (3.3).

The energetics for the non-resting basic state case are somewhat more complicated than the resting basic state case since energy can be extracted from the basic flow. From (2.8)-(2.10), with $F=Q=0$, we can obtain

$$
\frac{\partial}{\partial t} \int_{0}^{\infty} \frac{1}{2}\left(u^{2}+v^{2}+\phi^{2}\right) r d r+\int_{0}^{\infty} r u v \frac{d}{d r}\left(\frac{\bar{v}}{r}\right) r d r=0 .
$$

The last term in (9.4) represents a perturbation energy source term due to the integrated interaction of the Reynold's stress and the basic state tangential wind. Using (2.9) we can rewrite (9.4) as

$$
\frac{\partial}{\partial t} \int_{0}^{\infty} \frac{1}{2}\left(u^{2}+v^{2}+\phi^{2}\right) r d r=\frac{\partial}{\partial t} \int_{0}^{\infty}\left(\frac{\bar{\zeta}-\frac{2 \bar{v}}{r}}{1+\bar{\zeta}}\right) \frac{1}{2} v^{2} r d r \text {. }
$$

Using the definitions (4.4), (4.5) and

$$
c=\int_{0}^{\infty}\left(\frac{\bar{\zeta}-\frac{2 \bar{v}}{r}}{1+\bar{\zeta}}\right) \frac{1}{2}\left(v_{\infty}^{2}-v_{0}^{2}\right) r d r
$$

we can obtain from (9.5) the energy relation 


$$
\frac{K_{\infty}+P_{\infty}}{K_{0}+P_{0}}+\frac{\left(\begin{array}{c}
\text { gravity -inertia } \\
\text { wave energy }
\end{array}\right)}{K_{0}+P_{0}}=1+\frac{C}{K_{0}+P_{0}} .
$$

Thus, the final energy in the balanced flow $\left(K_{\infty}+P_{\infty}\right)$ and the energy in gravity-inertia wave motion come from the initial energy $\left(K_{0}+P_{0}\right)$ and the conversion from the basic state $(C)$.

As an example let us consider the basic state given by

$$
\begin{aligned}
& \bar{v}=F r \frac{4 \frac{r}{\bar{a}}}{\left(1+\frac{r^{2}}{\bar{a}^{2}}\right)^{2}}, \\
& \bar{\zeta}=\frac{8 F r}{\bar{a}} \frac{\left(1-\frac{r^{2}}{\bar{a}^{2}}\right)}{\left(1+\frac{r^{2}}{\bar{a}^{2}}\right)^{3}},
\end{aligned}
$$

where the Froude number $\mathrm{Fr}$ is the value of $\bar{v}$ at $r=\bar{a}$, the radius of vanishing relative vorticity. The basic state tangential wind $\bar{v}$ reaches a maximum at $r=\bar{a} / \sqrt{3}$ and the relative vorticity is slightly negative outside $r=\bar{a}$, as shown in Fig. 16. In the following we assume $F r=0.40$ and $\bar{a}=0.25$. Then, for an internal gravity wave speed of $40 \mathrm{~ms}^{-1}$ and $a$ Coriolis parameter corresponding to a latitude of $15.9^{\circ}$, the radius of vanishing relative vorticity is $250 \mathrm{~km}$, the radius of maximum wind is $144 \mathrm{~km}$, and the maximum wind is $21 \mathrm{~ms}^{-1}$. This is typical of a disturbance which has passed the tropical depression stage but still requires considerable intensification before becoming a hurricane.

As a perturbation on this basic state let us consider both the case where $v_{0}=0$ and $-\phi_{0}$ is given by the right hand side of $(6.1)$ and the case where $\phi_{0}=0$ and $v_{0}$ is given by (6.2). For any given horizontal scale of the initial perturbation, the final balanced state is obtained 
from the numerical solution of (3.4) subject to the boundary conditions (9.1) and (9.3), followed by numerical evaluation of (3.3). In this way we can construct energetic diagrams analogous to those of section 6 , where the basic state was assumed to ve at rest.

Fig. 17a summarizes the energetics associated with the case $v_{0}=0$ but $\phi_{0} \neq 0$, while Fig. 17b corresponds to the case $\phi_{0}=0$ but $v_{0} \neq 0$. In additicn to the final normalized geostrophic energy curves, we have included the normalized exchange of energy between the basic state and perturbation $\left(\frac{C}{K_{0}+P_{0}}\right)$. It is important to note that, because of this exchange, the normalized amount of energy which ends up in gravityinertia wave motion is now given by the difference between $\frac{K_{\infty}+P_{\infty}-C}{K_{0}+P_{0}}$ and unity.

From a qualitative point of view Figs. $17 \mathrm{a}$ and $17 \mathrm{~b}$ are similar to their resting basic state counterparts, Figs. $17 \mathrm{a}$ and $11 \mathrm{~b}$. That is, for smal1 scale rotational wind perturbations the efficiency of geostrophic energy generation is high, while smal1 scale pressure perturbations result in low geostrophic energy. For $a \geq 1$ the diagrams are barely modified by the presence of the non-resting basic state fields which we have chosen. But for $a<1$ there are some significant changes in the quantitative character of the energy curves. We see in Fig. 17a that the presence of the non-resting basic state increases the efficiency of the geostrophic energy generation for sma11 scale pressure perturbations. For example, when the basic state is at rest (Fig. 11a) and $a=0.2$, $K+P$

$\frac{\infty}{P_{0}}$ is only about $1 \%$, i.e. $99 \%$ of the energy escapes as gravityinertia wave motion: However, with the non-resting basic state we have chosen and $a=0.2, \frac{K_{\infty}+P_{\infty}}{P_{0}}$ is about $13 \%$. Thus, it would appear that, when a tropical disturbance acquires a significant relative vorticity 
field, convective heating within the region of positive relative vorticity can become much more efficient at producing balanced flow. The comparison of Fig. 17b with Fig. 11b also reveals significant quantitative differences. The directions of the changes found in this initial tangential wind case are generally opposite to those found in the initial pressure case. In addition, the magnitudes of the changes are generally larger. The large conversion of energy from the basic state to the perturbation for sma11 horizontal scales more than compensates for the loss of energy to gravity-inertia wave motion. This results in a $\frac{K_{\infty}+P_{\infty}}{K_{0}}$ which slightly exceeds unity for certain horizontal scales. 


\section{IMPLICATIONS FOR BOUNDARY CONDITIONS}

In the numerical simulation of a tropical cyclone using a primitive equation model one is forced to use a limited domain and hence to impose an outer boundary condition. There then arises the question of the distortion of the geostrophic adjustment process by the boundary condition. Although this problem is common to a11 limited area modeling with the primitive equations, it is particularly important in tropical cyclone models because the large amounts of latent heat released in convective clouds continually disrupt any approximate balance of pressure and wind and, as suggested in sections 4 and 6 , most of the latent heating is partitioned to gravity-inertia waves. The continual excitation of gravity-inertia waves by the heating patterns leads to the view that tropical cyclones must be highly radiating systems. Gravity-inertia waves falsely reflected back into the interior cause excessively high levels of ageostrophic motion. Although there are instances when this might be considered annoying but tolerable, in a moist model these spuriously reflected waves interact non-linearly with the moisture field, producing an erroneous modulation of the pattern of latent heat release.

It might be argued that dissipation can effectively remove these reflected waves before they reenter the region of interest so that the boundary condition is unimportant. A commonly used domain for tropical cyclone models is a region within a circle of about $1000 \mathrm{~km}$ radius. For such a domain size the transit times for waves with phase speeds of 200,100 and $50 \mathrm{~ms}^{-1}$ are $1.4,2.8$ and 5.6 hours respectively. Damping coefficients with damping times of onty a few hours seem excessively large. 
A thorough study of open boundary conditions for dispersive waves has been carried out by Bennett (1976). The application of Bennett's approach to the present problem proceeds as follows. Defining $\hat{\psi}(r, p)$ as the Laplace transform of $\psi(r, t)$, assuming the basic state is at rest, and assuming no initial disturbance and no forcing for $r \geq a$, we can transform (2.8)-(2.10) to obtain

$$
\begin{gathered}
p \hat{u}-\hat{v}+\frac{d \hat{\phi}}{d r}=0, \\
p \hat{v}+\hat{u}=0, \\
p \hat{\phi}+\frac{d r \hat{u}}{r d r}=0,
\end{gathered}
$$

for $r \geq a$. Eliminating $\hat{\phi}$ and $\hat{v}$ yields

$$
r^{2} \frac{d^{2} \hat{u}}{d r^{2}}+r \frac{d \hat{u}}{d r}-\left[\left(1+p^{2}\right) r^{2}+1\right] \hat{u}=0 \text { for } r \geq a \text {. }
$$

The solution which remains bounded as $r \rightarrow \infty$ is given by

$$
\hat{u}(r, p)=A(p) k_{1}\left[\left(1+p^{2}\right)^{\frac{1}{2}} r\right] \text { for } r \geq a \text {. }
$$

The transformed radial velocity component $\hat{u}$ satisfies

$$
\hat{u} \frac{d}{d r}\left\{r^{\frac{1}{2}} K_{1}\left[\left(1+p^{2}\right)^{\frac{1}{2}} r\right]\right\}-\frac{d r^{\frac{1}{2}} \hat{u}}{d r} k_{1}\left[\left(1+p^{2}\right)^{\frac{1}{2}} r\right]=0 \text { for } r \geq a .
$$

The exact outgoing condition is obtained by inverting (10.6). Aithough this can be done, the resulting expression is somewhat complicated. In order to illustrate the practical problems encountered with (10.6), let us use the asymptotic form of the modified Bessel function $k_{7}$, and simplify (10.6) to 


$$
\hat{u}+\left(7+p^{2}\right)^{-\frac{1}{2}} \frac{d r^{\frac{1}{2}} \hat{u}}{r^{\frac{1}{2}} d r}=0 \text { for } r \geq a \text {, }
$$

which can be inverted to give

$$
u(r, t)+\int_{0}^{t} J_{0}\left(t-t^{\prime}\right) \frac{\partial r^{\frac{1}{2}} u\left(r, t^{\prime}\right)}{r^{\frac{1}{2}} \partial r} d t^{\prime}=0 \text { for } r \geq a \text {. }
$$

Expressions of this type do not appear to be of great practical use since they require that we store and repeatedly sum (with different weights) boundary values of $u$ and $\frac{\partial u}{\partial r}$. In an approximate sense this procedure is equivalent to allowing the computational domain to expand in time, which is exactly what we are trying to avoid. Thus, in practice we may have to abandon the idea of using an exact outgoing boundary condition and be satisfied with something which gives low gravityinertia wave reflection.

In order to investigate the gravity-inertia wave reflectivity properties of various boundary conditions we begin by noting that the system (2.8) $-(2.10)$, with $\bar{v}=\bar{\zeta}=F=Q=0$, has the solution

$$
\left[\begin{array}{l}
u \\
v \\
\phi
\end{array}\right]=\left[\begin{array}{c}
1 \\
-\frac{j}{v} \\
-\frac{i k}{v}
\end{array}\right]\left\{\left[\begin{array}{l}
H_{1}^{(1)}(k r) \\
H_{1}^{(1)}(k r) \\
H_{0}^{(1)}(k r)
\end{array}\right]+R \cdot\left[\begin{array}{l}
H_{1}^{(2)}(k r) \\
H_{1}^{(2)}(k r) \\
H_{0}^{(2)}(k r)
\end{array}\right]\right\} e^{-i v t,}
$$

where $v=\left(1+k^{2}\right)^{\frac{1}{2}}, R$ is a complex constant, and $H_{v}^{(1)}$ and $H_{v}^{(2)}$ are the order $v$ Hankel functions of the first and second kind. Using the asymptotic expansions valid for large $\mathrm{kr}$ we can write

$$
\left(\begin{array}{l}
u \\
v \\
\phi
\end{array}\right) \sim\left(\frac{2}{\pi k r}\right)^{\frac{1}{2}} e^{-\frac{3 \pi i}{4}}\left(\begin{array}{c}
T \\
-\frac{i}{v} \\
\frac{k}{v}
\end{array}\right)\left\{e^{j(k r-v t)}-i R e^{-i(k r+v t)}\right\}
$$


Hence, the first term of (10.9) and (10.10) corresponds to an outgoing wave and the second term to an incoming wave.

If a denotes the radius of the boundary, the asymptotic form of the outgoing wave satisfies

$$
\frac{\partial u}{\partial t}+\frac{v}{k} \frac{\partial r^{\frac{1}{2}} u}{r^{\frac{1}{2}} \partial r}=0 \text { at } r=a \text {, }
$$

with similar expressions for $v$ and $\phi$. Equation (I) should serve as a fairly accurate radiation condition. Since $\frac{\nu}{k} \rightarrow 1$ as $k \rightarrow \infty$ (I) might be approximated by

$$
\frac{\partial u}{\partial t}+\frac{\partial r^{\frac{1}{2}} u}{r^{\frac{1}{2}} \partial r}=0 \text { at } r=a \text {, }
$$

or by

$$
\frac{\partial u}{\partial t}+\frac{\partial \cdot \lambda}{\partial r}=0 \text { at } r=a
$$

Two other boundary conditions in common use are

$$
\frac{\partial r u}{r \partial r}=0 \text { at } r=a \text {, }
$$

and

$$
u=0 \text { at } r=a \text {. }
$$

The reflectivities of conditions (I) - (V) can be found by substituting (10.9) and solving for $|R|$. The results are shown in Table 1 and Fig. 20. Boundary condition II appears to give reflectivities nearly as low as $I$. In addition it has the advantage of using the pure gravity wave speed (unity in this case) for $v / k$.

A survey of the Titerature on tropical cyclone models indicates that the boundary conditions used are either type IV (Rosenthal, 1970; 


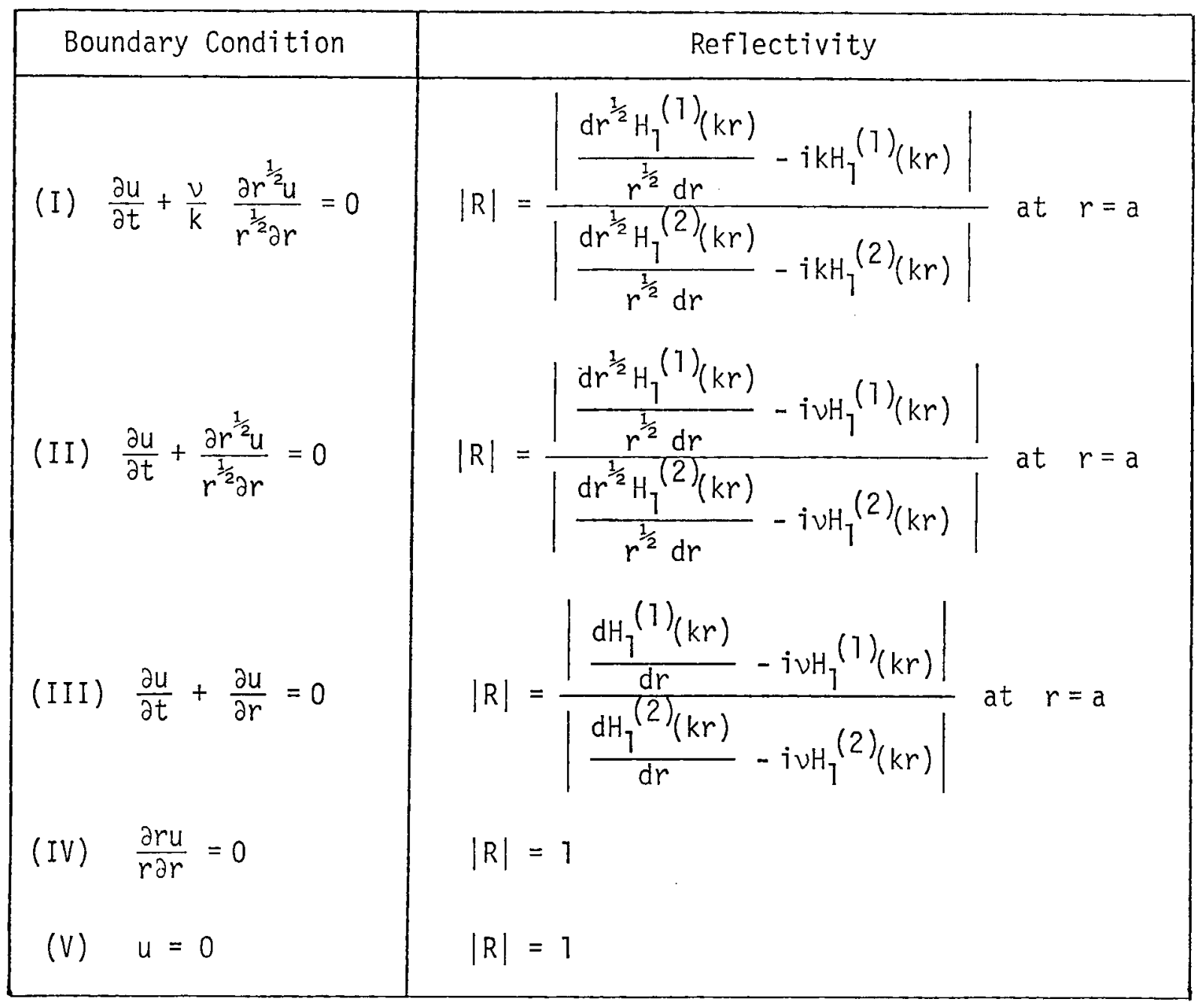

Table 1. Reflectivities for the various boundary conditions. 
Anthes, 1971, 1977; Anthes et a1., 1971a,b) or type V (Yamasaki, 1968a; Kurihara, 1975; Kurihara and Tuleya, 1974; Rosentha1, 1978). Rosenthal (1971) has compared the ultimate intensity attained by model cyclones with either boundary conditions IV or $V$ as a function of the size of the computational domain. For domain sizes less than $2000 \mathrm{~km}$ there are substantial differences, with boundary condition $V$ resulting in less intense model cycicnes. Rosenthal's interpretation is that boundary condition $V$, coupled with a small domain size, forces upper level outflow air to subside too strongly and too near the storm center, resulting in an unrealistic decrease in the radial temperature gradient. The analysis presented in this paper would indicate that neither IV nor $V$ is very satisfactory from the geostrophic adjustment point of view.

The generalization of boundary condition (II) to the fully stratified case involves the solution of the vertical structure problem for its eigenvalues $\left(h_{n}\right)$ and eigenfunctions. The dimensional form of the boundary condition (with the factor $\sqrt{g h_{n}}$ in front of the second term) is then applied to each vertical mode. Some comparison tests which we have made indicate that boundary condition (II) results in less distorticn of the adjustment process than the numerical extrapolation technique of Orlanski (1976), which is, however, somewhat easier to implement. The stratified version of boundary condition (II) is presently being used in an axisymmetric tropical cyclone model developed at colorado State University. 


\section{CONCLUDING REMARKS}

It has been shown that there exist simple analytical solutions for the geostrophic adjustment of an axisymmetric vortex. The examples discussed above indicate that, when the basic flow is at rest, the energetical efficiency of cloud cluster heating in producing balanced vortex flow is very low while the efficiency of cloud cluster forcing of the vorticity field is very high. When the basic flow is not at rest, important modifications of these energy partition relations can occur.

In tropical cloud cluster budget studies it is common practice to compute either the apparent heat source or the apparent vorticity source. The results presented here emphasize that the effect of clouds on the potential vorticity field (i.e. the apparent potential vorticity source) is the important ingredient in understanding the feedback of clouds on the large-scale fields.

Because gravity-inertia waves can be so strongly excited by heating on horizontal scales small compared to the radius of deformation, the lateral boundary condition becomes an important factor in the ability of a primitive equation tropical cyclone model to simulate geostrophic adjustment. Although not perfect, a suitable radiation condition may be sufficient for most purposes.

There are several ways to generalize the results presented here. First of a 11, the effects of stratification could be more throughly studied by considering all the vertical modes rather than just the first internal mode, as we have done here. This has been done to a certain extent by Bolin (1953), Kibel $(1955,1957,1963)$, Fjeisted (1958), Monin (1958) and Fischer (1963), although not with axisymmetric tropical 
disturbances in mind. Secondly, the f-ptane assumption could be retained but the axisymmetric assumption relaxed, and a Fourier representation used in the tangential direction. Then, using Hankel transforms whose. orcer is determined by the azimuthal wavenumber, many of the results presented here can be easily generalized. This asymmetric adjustment problem will be discussed in a future paper. Thirdly, curvature effects could be included and differences between geostrophic and gradient adjustment studied. However, gradient adjustment is less amenable to theoretical analysis. Finally, in order to study the adjustment process very near the equator, it is preferable to abandon the f-plane in favor of the equatorial $\beta$-plane. Then, an unbalanced initial state can be projected onto the eigenfunctions of the equatorial $\beta$-plane and the subsequent flow patterns studied. This has been done by Silva Dias and Schubert (1979), who show that the energy partition relations derived on the f-plane are qualitatively valid if the energy of the final geostrophic state is now interpreted as the energy in Rossby wave motion. However, there are important differences between the $f-p l$ ane and $\beta$-plane results. For example, if the unbalanced initial state has circular symmetry, gravity-inertia wave dispersion quickly leaves behind a quasibalanced symmetric flow which is then slowly distorted by the Rossby wave dispersion process. 


\section{ACKNOWLEDGEMENTS}

The authors are grateful to Drs. Duane Stevens, William Gray, William Blumen, Jan Paegle and Julia Paegle for their valuable comments on this work. We are also indebted to Odilia Panella for her help in preparing the manuscript. The research reported here has been jointly supported by NSF and NOAA through NSF Grant ATM-7808125. Acknowledgement is a lso made to the National Center for Atmospheric Research, which is sponsored by the National Science Foundation, for computer time used in this research. 


\section{REFERENCES}

Anthes, R.A., 1971: A numerical model of the slowly varying tropical cyclone in isentropic coordinates. Mon. Wea. Rev., 99, 617-635.

, 1972: Development of asymmetrics in a three-dimensional

numerical mndel of the tropical cyclone. Mon. Wea. Rev., 100, 461-476.

, 1977: Hurricane model experiments with a new climulus parameterization scheme. Mon. Wea. Rev., 105, 287-300.

, S.L. Rosenthal and J.W. Trout, 1971a: Preliminary results from an asymmetric model of the tropical cyclone. Mon. Wea. Rev., 99, 744-758.

, J.W. Trout and S.L. Rosenthal, 1971b: Comparisons of tropical cyclone simulations with and without the assumption of circular symmetry. Mon. Wea. Rev., 99, 759-766.

Arakawa, A., and V.R. Lamb, 1977: Computational design of the basic dynamical processes of the UCLA general circulation model. Methods in Computational Physics, 17, 173-265.

Bennett, J.R., 1976: Open boundary conditions for dispersive waves. J. Atmos. Sci., 33, 176-182.

B7umen, W., 1967: On non inear geostrophic adjustment. J. Atmos. Sci., 24, 325-332.

1972: Geostrophic adjustment. Rev. Geophys. Space Phys., 10, $485-528$.

, and W.M. Washington, 1969: The effect of horizontal shear flow on geostrophic adjustment in a barotropic fluid. Tellus, 21, 167176.

Bolin, B., 1953: The adjustment of a nonbalanced velocity field towards geostrophic equilibrium in a stratified fluid. Tellus, 5, 373-385.

Cahn, A., 1945: An investigation of the free oscillations of a simple current system. J. Meteoro1., 2, 113-119.

Chu, J.-H., 1976: Vorticity in maritime cumulus clouds and its effects on the large-scale budget of vorticity in the tropics. Ph.D. Thesis, UCLA.

Dobrischman, E.M., 1964: On Coriolis force variability in the prognostic schemes. Tellus, 16, 18-25.

Erdelyi, A., W. Magnus, F. Oberhettinger, and F.G. Tricomi, 1954: Tables of Integral Transforms, 2 volumes. McGraw-Hill, New York.

Fischer, G., 1963: ̈̈ber die Adaption einfacher Stromfelder in der Atmosphäre. Ber. Dent. Wetterdienstes, 12(87), 87/3-87/20. (Engl. transl. available from $W$. Schubert, Dept. of Atmospheric Science, Colorado State University.) 


\section{REFERENCES continued}

Fjelsted, J.E., 1958: Ocean current as an initial value problem. Geophys. Pub7., 20(7), 24pp.

Geisler, J.E., 1970: Linear theory of the response of a two-layer ocean to a moving hurricane. Geophys. Fluid Dyn., 1, 249-272.

, and R.E. Dickinson, 1972: The role of variable Coriolis parameter in the propagation of inertia-gravity waves during the process of geostrophic adjustment. J. Phys. Oceanogr., 2, 263-272.

Hodur, R.M., and J.S. Fein, 1977: A vorticity budget over the Marsha11 Islands during the spring and summer months. Mon. Wea. Rev., 105, $1521-1526$.

Janjic, Z., and A. Wiin-Nielson, 1977: On geostrophic adjustment and numerical procedures in a rotating fluid. J.Atmos. Sci., 34, 297370 .

Kibel, I.A., 1955: On the adaption of air motion to the geostrophic law. Dok1. Akad. Sci. SSSR, 104, 60-63. (Eng1. trans. available from W. Schubert, Dept. of Atmospheric Science, Colorado State (Iniversity.)

, 1957: The analysis of geostrophic motion in weather prediction. Trans. Cent. Inst. Forecasting (Moscow), No. 60, 3-9. (Engl. trans. available from W. Schubert, Dept. of Atmospheric Science, Colorado State University.)

, 1963: An Introduction to the Hydrodynamical Methods of Short Period Weather Forecasting. Translation edited by R. Baker. Pergamon, New York, 375pp.

Kuo, H.L., 1965: On formation and intensification of tropical cyclones through latent heat release by cumulus convection. J. Atmos. Sci., $22,40-63$.

Kurihara, Y., 1975: Budget analys is of a tropical cyclone simulated in an axisymmetric numerical model. J. Atmos. Sci., 32, 25-59.

, and R.E. Tuleya, 1974: Structure of a tropical cyclone developed in a three dimensional numerical simulation model. J. Atmos. Sci., 31, 893-919.

Madala, R.V., and S.A. Piacsek, 1975: Numerical simulation of asymmetric hurricanes on a $\beta$-plane with vertical shear. Tel7us, 27, 453-468.

Mathur, M.B., 1974: A multi-grid primitive equation model to simulate the development of an asymmetric hurricane (Isebel1, 1964). J. Atmos. Sci., 31, 371-393.

Miles, J.W., 1971: Integral Transforms in Applied Mathematics. Cambridge University Press, 97pp. 
REFERENCES continued

Monin, A.S., 1958: Pressure variations in a baroclinic atmosphere. Izv. Acad. Sci. USSR, Phys. Solid Earth, No. 4, 280-286.

Obukhov, A.M., 1949: On the question of the geostrophic wind (in Russian), Izv. ikad. Nauk SSSR Ser. Geograf.-Geofiz., 13(4), 281-306. (Eng1. trans1. available from W. Schubert, Dept. of Atmospheric Science, Colorado State University.)

Ogura, Y., 1964: Frictionally controled, thermal1y driven circulation in a circular vortex with application to tropical cylones. J. Atmos. Sci., 21, 610-621.

, and H.-R. Cho, 1973: Diagnostic determination of cumulus cloud populations from observed large-scale variables. J. Atmos. Sci., 30, $1276-1286$.

Ooyama, K., 1969a: Numerical simulation of the life cycle of tropical cyclones. J. Atmos. Sci., 26, 3-40.

, 1969b: Numerical simulation of tropical cyclones with an axisymmetric model. Proceedings of the WMO/IUGG Symposium on Numerical Weather Prediction, Tokyo, 1968. III:81-88.

Orlanski, I., 1976: A simple boundary condition for unbounded hyperbolic flows. 3. Computational Physics, 21, 251-269.

Paegle, J., 1978: The transient mass-flow adjustment of heated atmospheric circulation. J. Atmos. Sci., 35, 1678-1688.

Phillips, N.A., W. Blumen, and O. Cote', 1960: Numerical weather prediction in the Soviet Union. Bu11. Amer. Meteorol. Soc., 41, 599-617.

Reed, R.J., and E.E. Recker, 1971: Structure and properties of synopticscale wave disturbances in the equatorial western Pacific. J. Atmos. Sci., 28, 1117-1133.

, and R.H. Johnson, 1974: The vorticity budget of synoptic-scale wave disturbances in the tropical western Pacific. J. Atmos. Sci., 31, 1784-1790.

, D.C. Norquist and E.E. Recker, 1977: The structure and properties of African wave disturbances as observed during Phase III of GATE. Mon. Wea. Rev., 105, 317-333.

Richtmyer, R.D., and K.W. Morton, 1967: Difference Methods for Initialvalue Problems. New York, Interscience Publishers, 198-201.

Rosenthal, S.L., 1970: A circularly symmetric primitive equation model of tropical cyclone development containing an explicit water vapor cycle. Mon. Wea. Rev., 98, 643-663. 


\section{REFERENCES continued}

Rosenthal, S.L., 1971: The response of a tropical cyclone model to variations in boundary parameters, initial conditions, lateral boundary conditions, and domain size. Mon. Wea. Rev., 99, 767-777.

, 1978: Numerical simulation of tropical cyclone development with latent heat release by the resolvable scales, I: model description and preliminary results. J. Atmos. Sci., 35, 258-271.

Rossby, C.-G., 1938: On the mutual adjustment of pressure and velocity distributions in certain simple current systems, 2. J. Mar. Res., I, 239-263.

Ruprecht, E., and W.M. Gray, 1976: Analys is of satellite-observed tropical cloud clusters. I. Wind and dynamic fields. Tellus, 28, $391-413$.

Schoenstadt, A.L., 1977: The effect of spacial discretization on the steady-state and transient solutions of a dispersive wave equation. J. Comput. Phys., 23, 364-379.

, 1979: On one-dimensional geostrophic adjustment with finite differencing. Mon. Wea. Rev., 107, 211-214.

- 1980: A transfer function analysis of numerical schemes used to simulate geostrophic adjustment. Submitted to Mon. Wea. Rev.

Shapiro, L.J., 1978: The vorticity budget of a composite African tropical wave disturbance. Mon. Wea. Rev., 106, 806-817.

Silva Dias, P.L., and W.H. Schubert, 1979: The dynamics of equatorial mass-flow adjustment. Atmospheric Science Paper No. 312, Department of Atmospheric Science, Colorado State University, Fort Collins, Colorado. 203 pages.

Stevens, D.E., 1979: Vorticity, momentum and divergence budgets of synoptic-scale wave disturbances in the tropical eastern Atlantic. Mon. Wea. Rev., 107, 535-550.

Sundquist, H., 1970a: Numerical simulation of the development of tropical cyclones with a ten-level model, part I. Tellus, 22, 359-390.

, 1970b: Numerical simulation of the development of tropical cyclones with a ten-level model, part II. Tellus, 22, 504-510.

Thompson, R.M., S.W. Payne, E.E. Recker and R.J. Reed, 1979: Structure and properties of synoptic-scale wave disturbances in the Intertropical Convergence Zone of the eastern Atlantic. J. Atmos. Sci., 36, 53-72. 
REFERENCES continued

Veronis, G., 1956: Partition of energy between geostrophic and nongeostrophic oceanic motions. Deep-Sea Res., 8, 157-177.

Williams, K.T., and W.M. Gray, 1973: Statistical analysis of satelliteobserved trade wind cloud clusters in the western North Pacific. Te11us, 25, 313-336.

Yamasaki, M., 1968a: Numerical simulation of tropical cyclone development with the use of primitive equations. J. Meteor. Soc. Japan, 46, 178201.

1968b: Detailed analys is of a tropical cyclone simulated with a 13-Tayer mode1. Papers in Meteorology and Geophysics, 19, 559585 .

Yanai, M., S. Esbensen and J.-H. Chu, 1973: Determination of bulk properties of tropical cloud clusters from large-scale heat and moisture buidgets. J. Atmos. Sci., 30, 611-627.

J.-H. Chu, T.E. Stark and T. Nitta, 1976: Response of deep and shallow tropical maritime cumuli to large-scale processes. J. Atmos. Sci., 33, 976-991. 


\section{LIST OF FIGURES}

Figure 1. Weighting functions $\frac{1}{1+k^{2}}$ and $\frac{k^{2}}{1+k^{2}}$. See text for discussion.

Figure 2. Normalized initial geopotential perturbation (see Eq. (5.2)) and final adjusted geopotential for the initial top-hat potential vorticity perturbation. The three final states correspond to initial perturbations with horizontal scales of $a=0.2,1.0$, and 5.0 .

Figure 3. Normalized initial tangential velocity perturbation (see Eq. (5.3)) and final adjusted tangential velocity for the initial top-hat potential vorticity perturbation. The three final states correspond to initial perturbations with horizontal scales of $a=0.2,1.0$ and 5.0 .

Figure 4. Normalized initial vorticity perturbation (interpreted according to Eq. (5.1), $\phi_{0}=0$ ) and final adjusted vorticity for the initial top-hat potential vorticity perturbation. The three final states correspond to initial perturbations with horizontal scales of $a=0.2,1.0$, and 5.0 .

Figure 5. (a) Ratios of the final perturbation energies in geostrophic flow to the initial perturbation available potential energy (top-hat geopotential perturbation) as a function of the horizontal scale of the initial perturbation. The basic state is at rest. The distance of the curves below unity indicates the fraction of the initial perturbation energy partitioned to gravity-inertia wave motion.

(b) Same as (a) except that the initial perturbation is in the tangential velocity field. 
Figure 6 . Isolines of radial mass flux $r u(r, t)$ in the $(r, t)$ plane for the case where the initial vorticity is concentrated at the origin and the initial geopotential field is flat. The contour interval is 0.5 with dashed lines indicating negative $r u(r, t)$.

Figure 7. Divergence, $\frac{\partial r u}{r \partial r}$, as a function of time for $r=\pi / 4$ and $r=3 \pi / 4$. The initial condition is the same as for Fig. 6 .

Figure 8 . The geopotential surface $\phi(r, t)$ in the $(r, t)$ plane for the same initial condition as in Figs. 6 and 7 . This may also be interpreted as the vorticity since in this case $\zeta(r, t)=$ $\phi(r, t)$ for all $r \neq 0$.

Figure 9. Normalized plots of the final adjusted geopotential fields (dashed) as contrasted with the initial geopotential fields (solid) where the initial geopotential fields represent Gaussian-type potential vorticity perturbations. Curves are plotted for perturbations with horizontal scales of $a=0.2$ and 5.0 .

Figure 10. Normalized plots of the final adjusted tangential velocity fields (dashed) as contrasted with the initial tangential velocity fields (solid) where the initial tangential velocity fields represent Gaussian-type potential vorticity perturbations. Curves are piotted for perturbations with horizontal scales of $a=0.2$ and 5.0 . 
Figure 11. (a) Ratios of the final perturbation energies in geostrophic flow to the initial perturbation available potential energy (Gaussian-type geopotential perturbation) as a function of the horizontal scale of the initial peiturbation. The basic state is at rest. The distance of the total energy curve below unity indicates the fraction of the initial perturbation energy partitioned to gravity-inertia wave motion.

(b) Same as (a) except that the initial perturbation is in the tangential velocity field (Eq. (6.2)).

Figure 12. Isolines of radial mass flux $r u(r, t)$ in the $(r, t)$ plane for the case where the initial divergence is concentrated near the origin, the initial tangential velocity is everywhere zero, and the initial geopotential field is flat. The contour interval is 0.5 with dashed lines indicating negative $r u(r, t)$.

Figure 13. Divergence, $\frac{\partial r u}{r \partial r}$, as a function of time for $r=\pi / 4$ and $r=3 \pi / 4$. The initial condition is the same as for Fig. 12 .

Figure 14. The geopotential surface $\phi(r, t)$ in the $(r, t) p l a n e$ for the same initial condition as in Figs. 13 and 14 . This may also be interpreted as the vorticity since in this case $\zeta(r, t)=$ $\phi(r, t)$ for all $r$.

Figure 15. The geopotential surface $\phi(r, t)$ in the $(r, \alpha t)$ plane for the case of (a) the balanced model, (b) the primitive equation model with slow forcing $\left(\alpha=\frac{1}{2}\right)$ and $(c)$ the primitive equation model with rapid forcing $(\alpha=2)$. 
Figure 16. Plots of the basic state quantities $\bar{v},\left(1+\frac{2 \bar{v}}{r}\right)$ and $(1+\bar{\zeta})$, as function of $r / \bar{a}$, for the case $\bar{a}=0.25$ and $\mathrm{Fr}=0.40$. The dimensional scales, included for convenience, are based on an inte: nal gravity wave speed of $40 \mathrm{~ms}^{-1}$ and a Coriol is parameter corresponding to a latitude of $15.9^{\circ}$.

Figure 17. (a) Ratios of the final perturbation energies in balanced flow to the initial perturbation available potential energv (Gaussian-type geopotential perturbation) as a function of the horizontal scale of the initial perturbation. The basic state, which is not at rest, was shown in Fig. 17. A curve showing the ratio of the energy exchange with the basic state to the initial available potential energy is also included $\left(C / P_{0}\right)$. The distance of the $\frac{P_{\infty}+K_{\infty}-C}{P_{0}}$ curve below unity indicates the fraction of initial perturbation available potential energy which ends up in gravity-inertia wave motion. (b) Same as (a) except that the initial perturbation is in the tangential velocity field (Eq. (6.2)).

Figure 18. Plots of wave reflectivities for the boundary conditions presented in Table 1. Unity indicates perfect reflection. The abscissa can be read in either ka (wavenumber in units of domain size) or $2 \pi / k a$ (wavelength in units of domain size). 


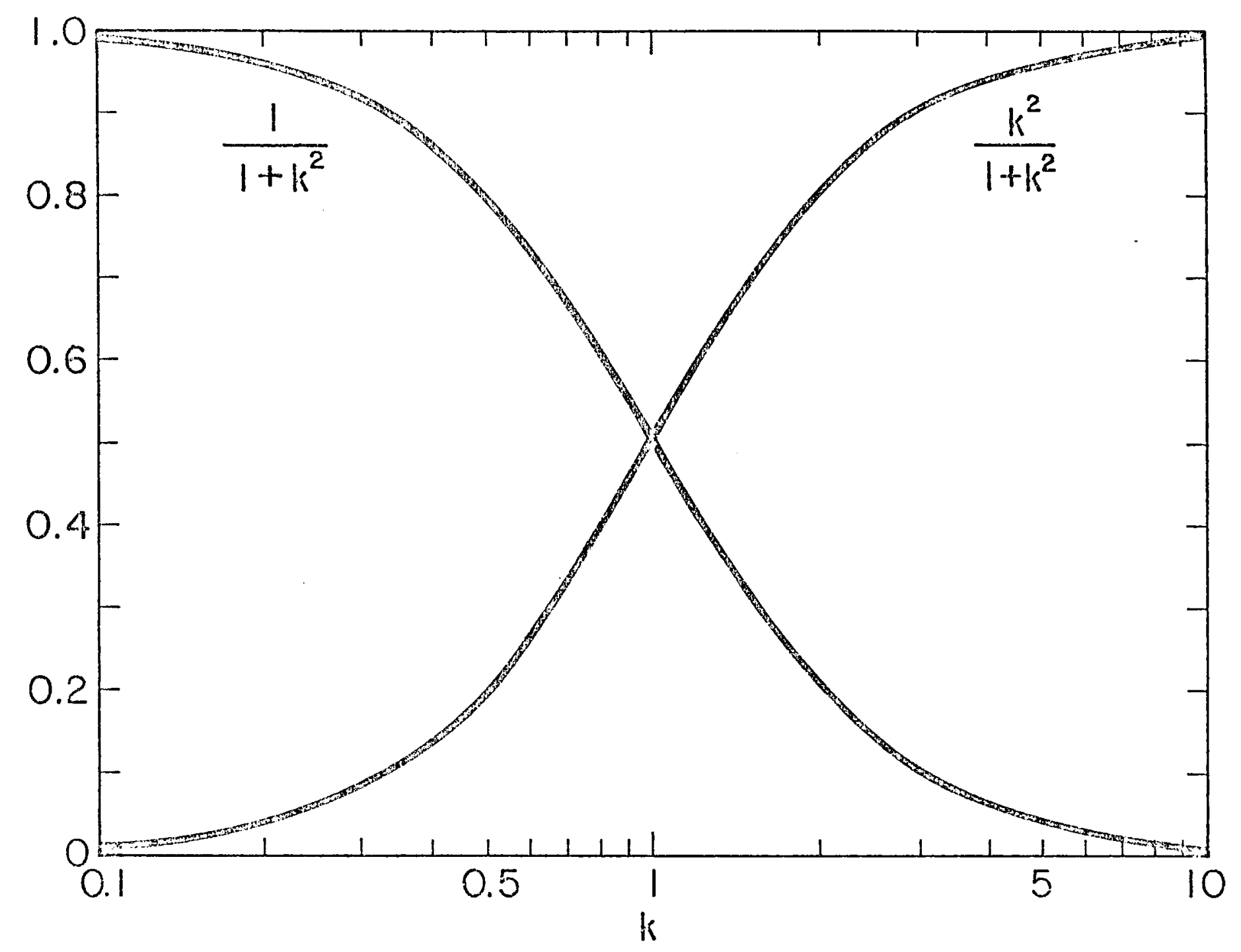

Figure 1. 


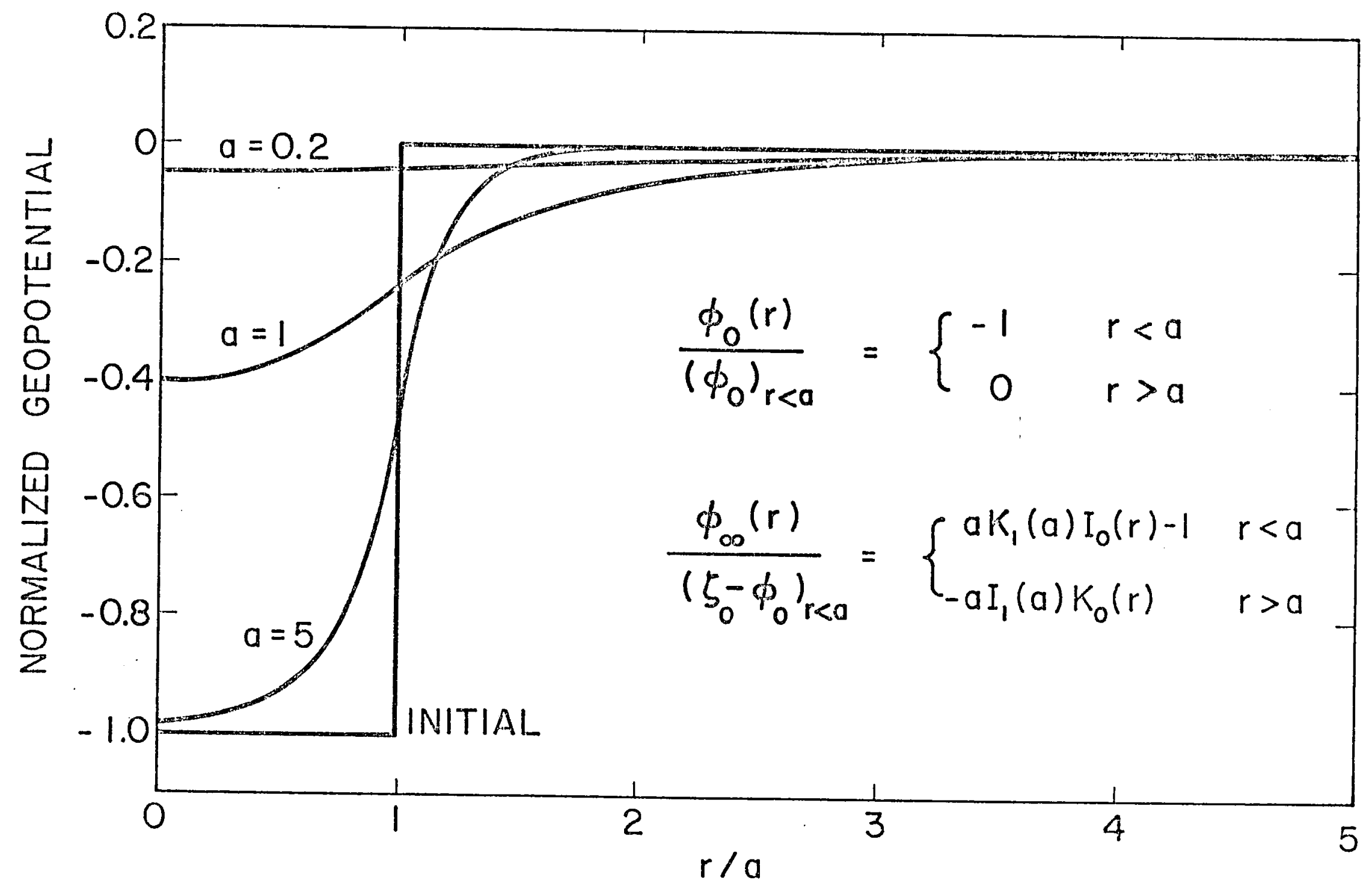

Figure 2. 


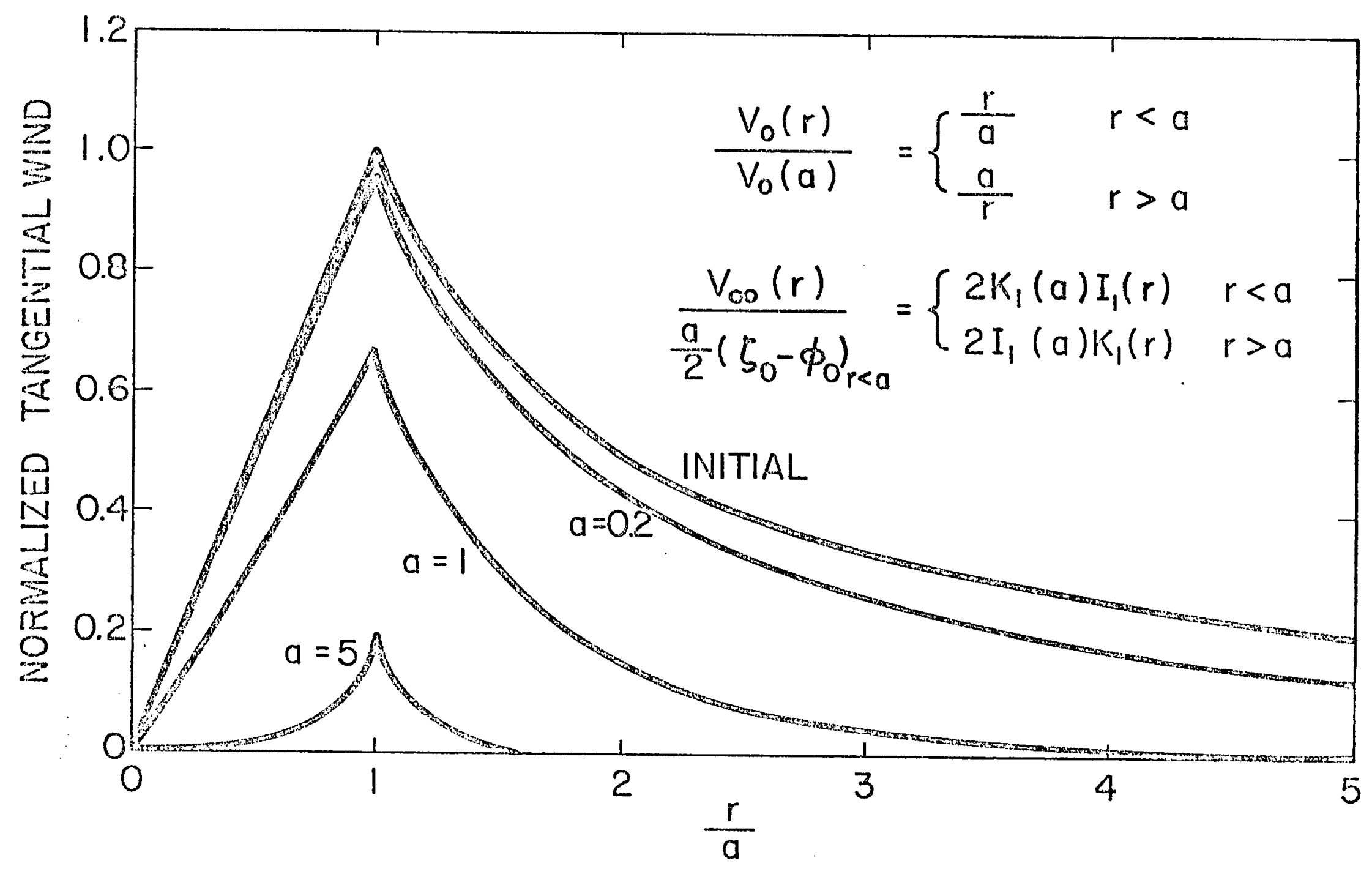

Figure 3. 


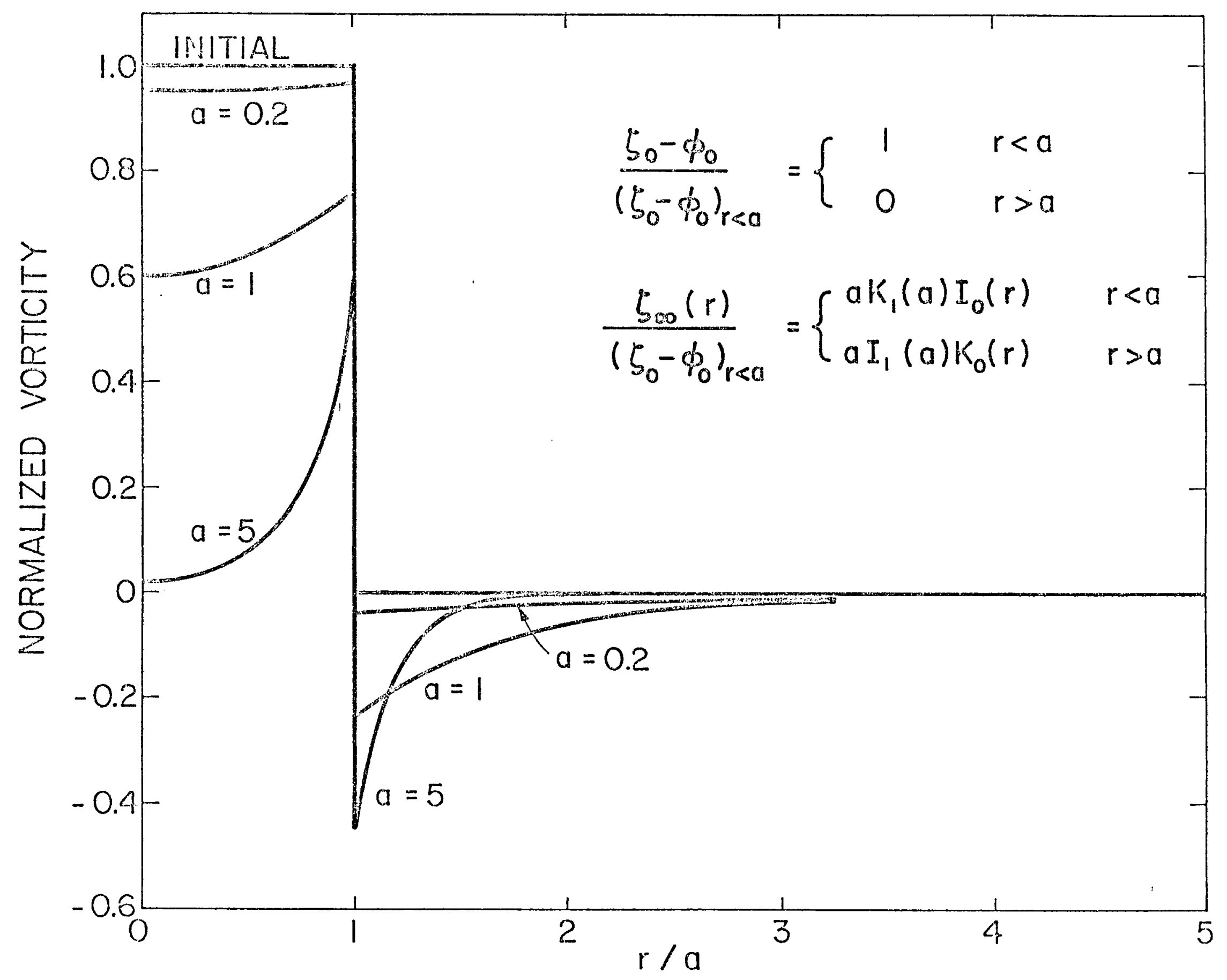

Finure 4. 


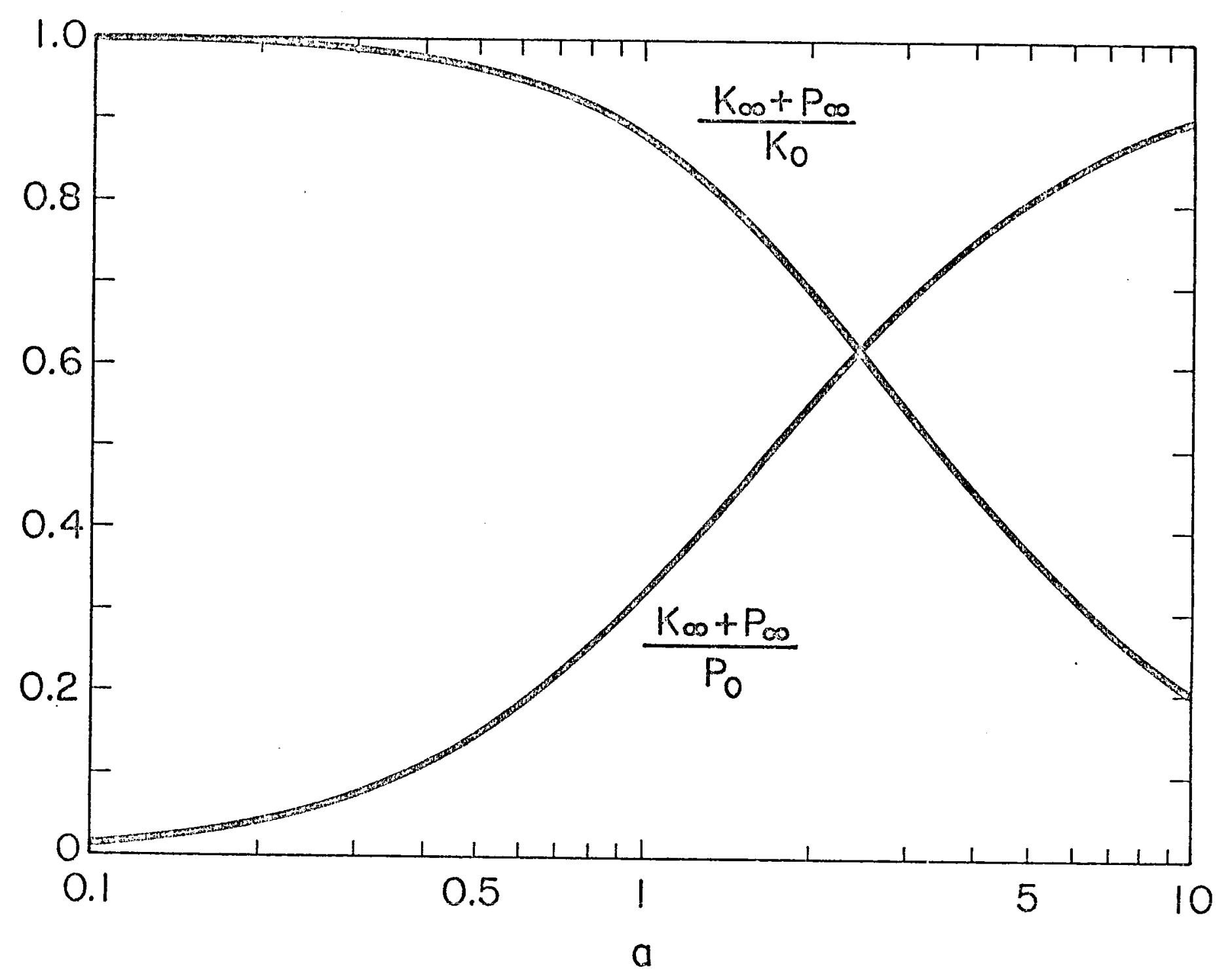

Figure 5a. 


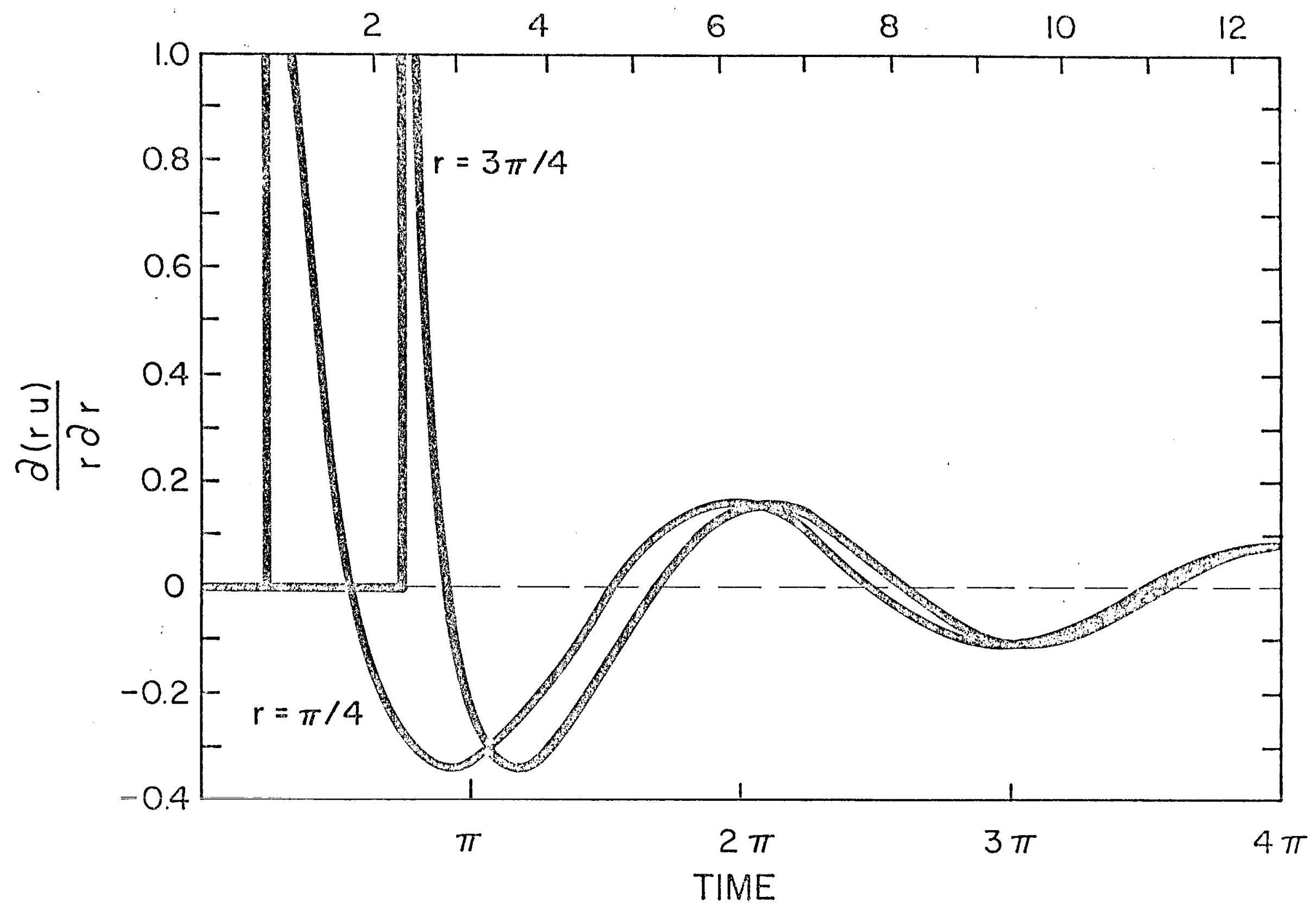




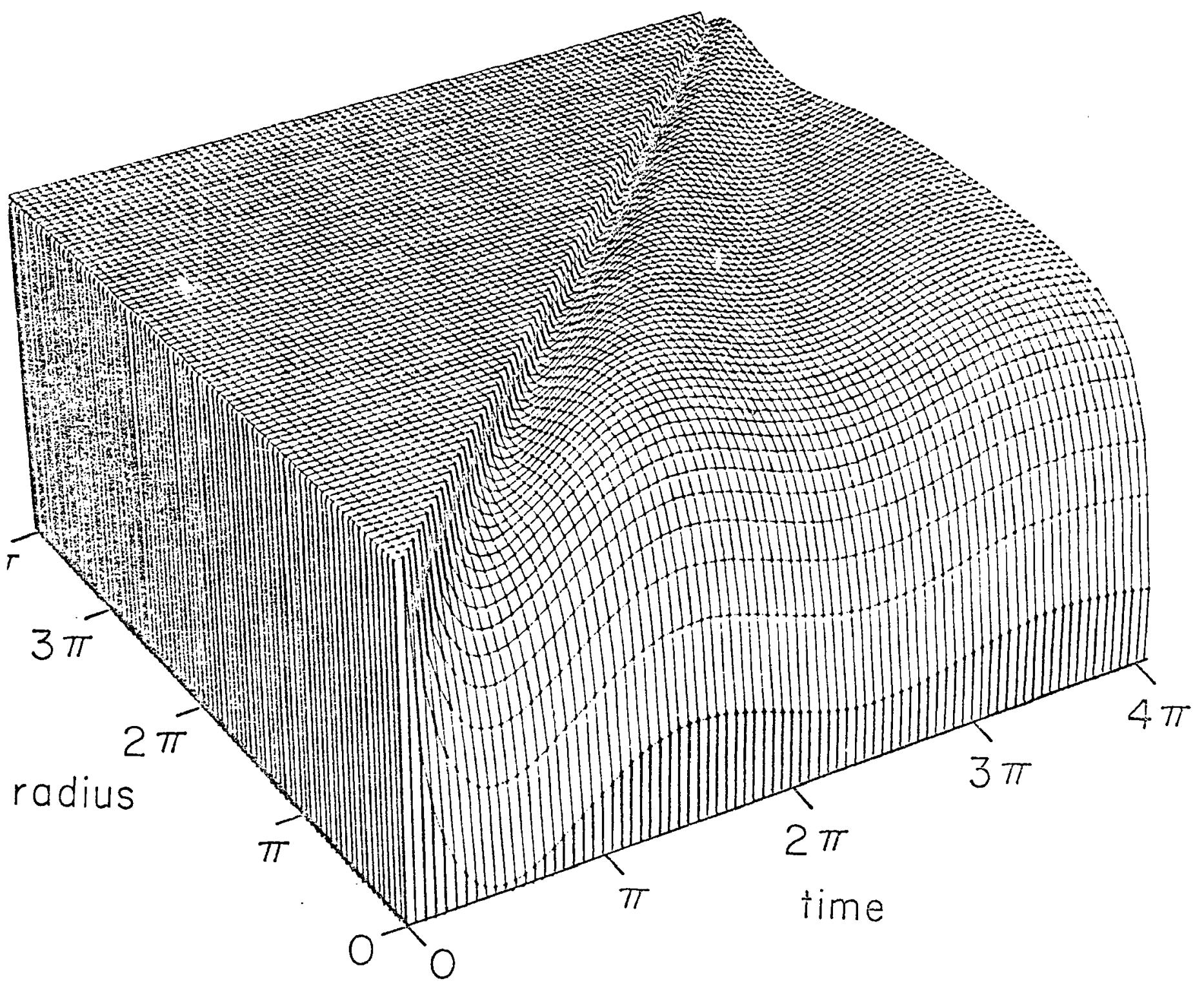

Figure 8. 


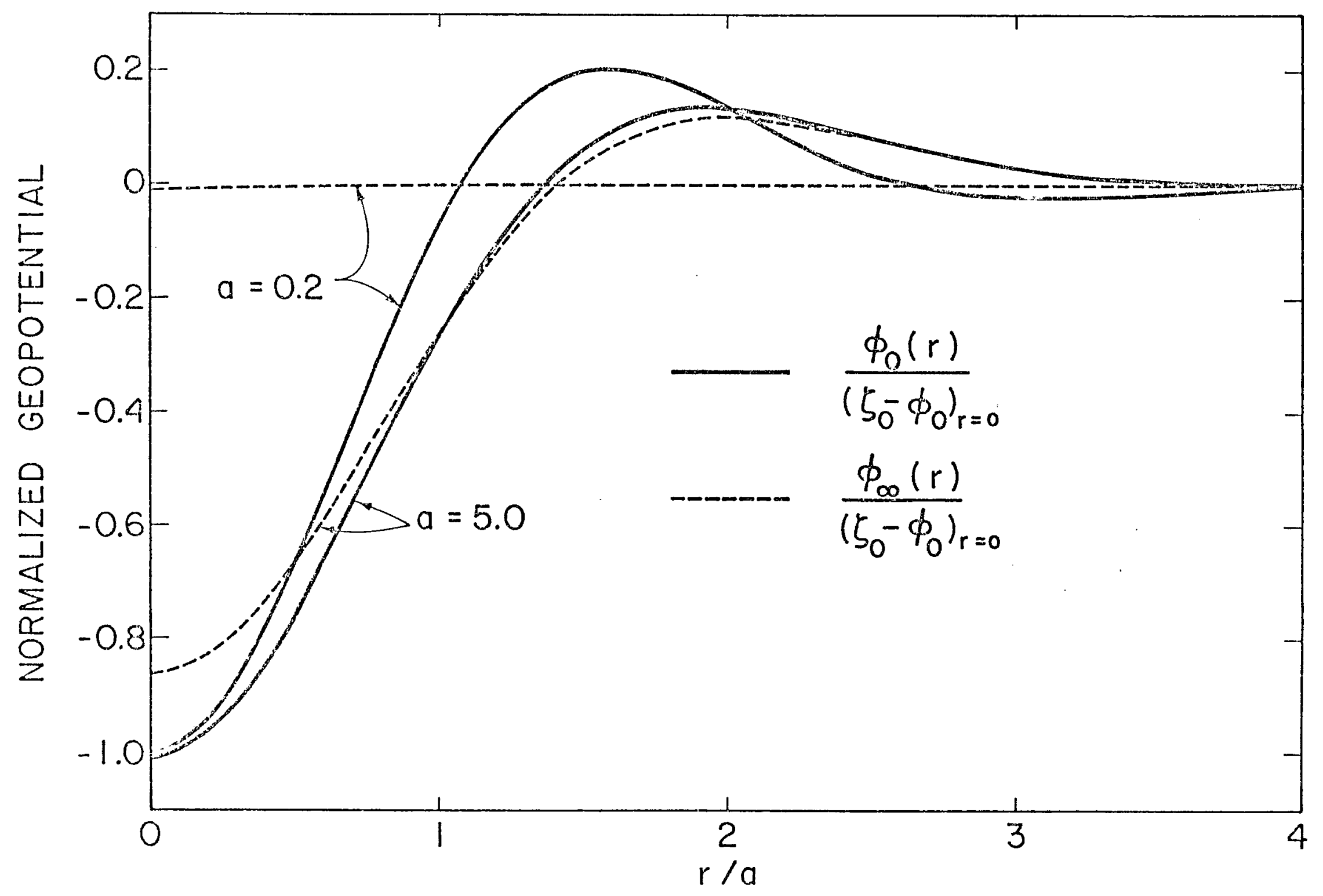

Figure 9. 


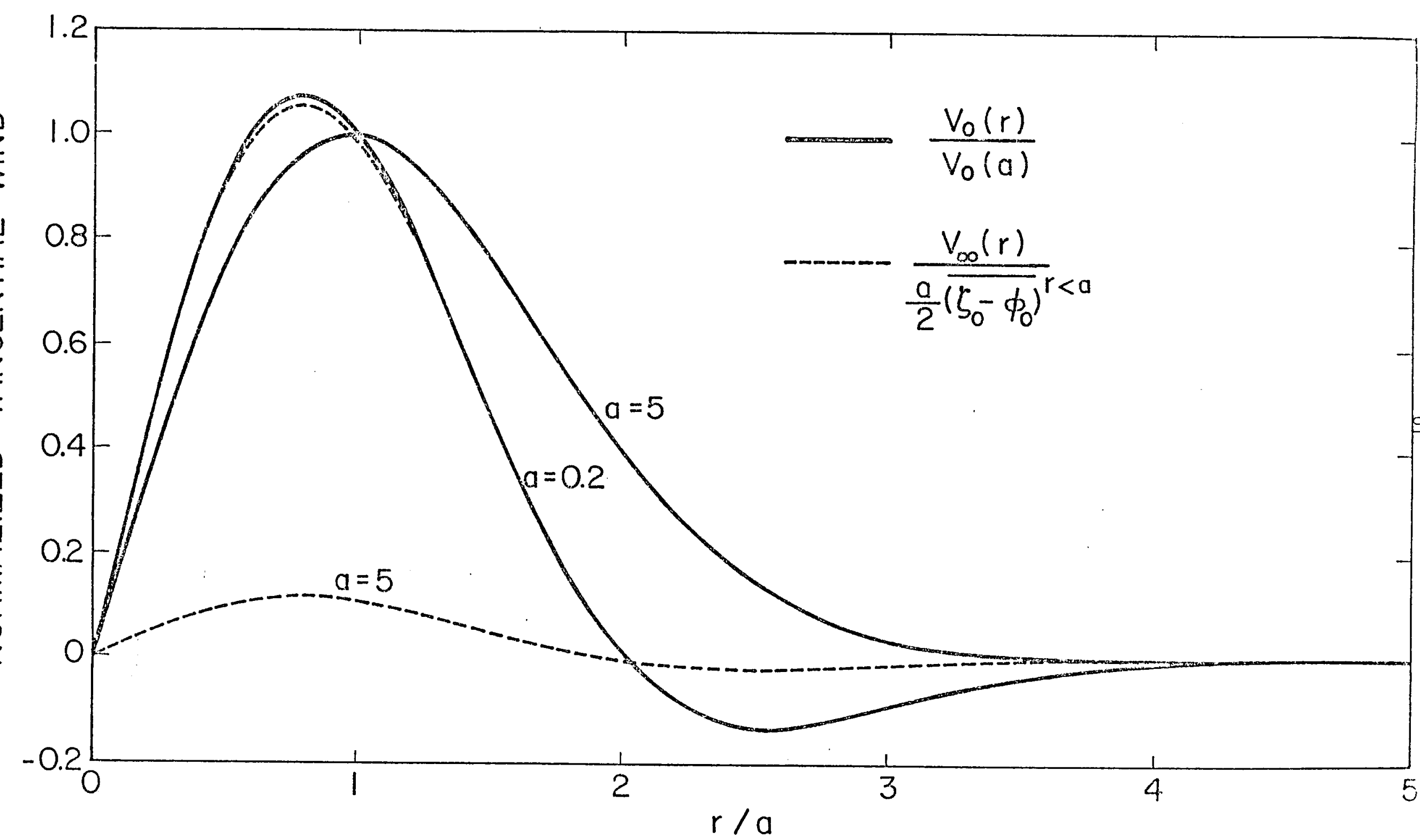

Figure 10. 


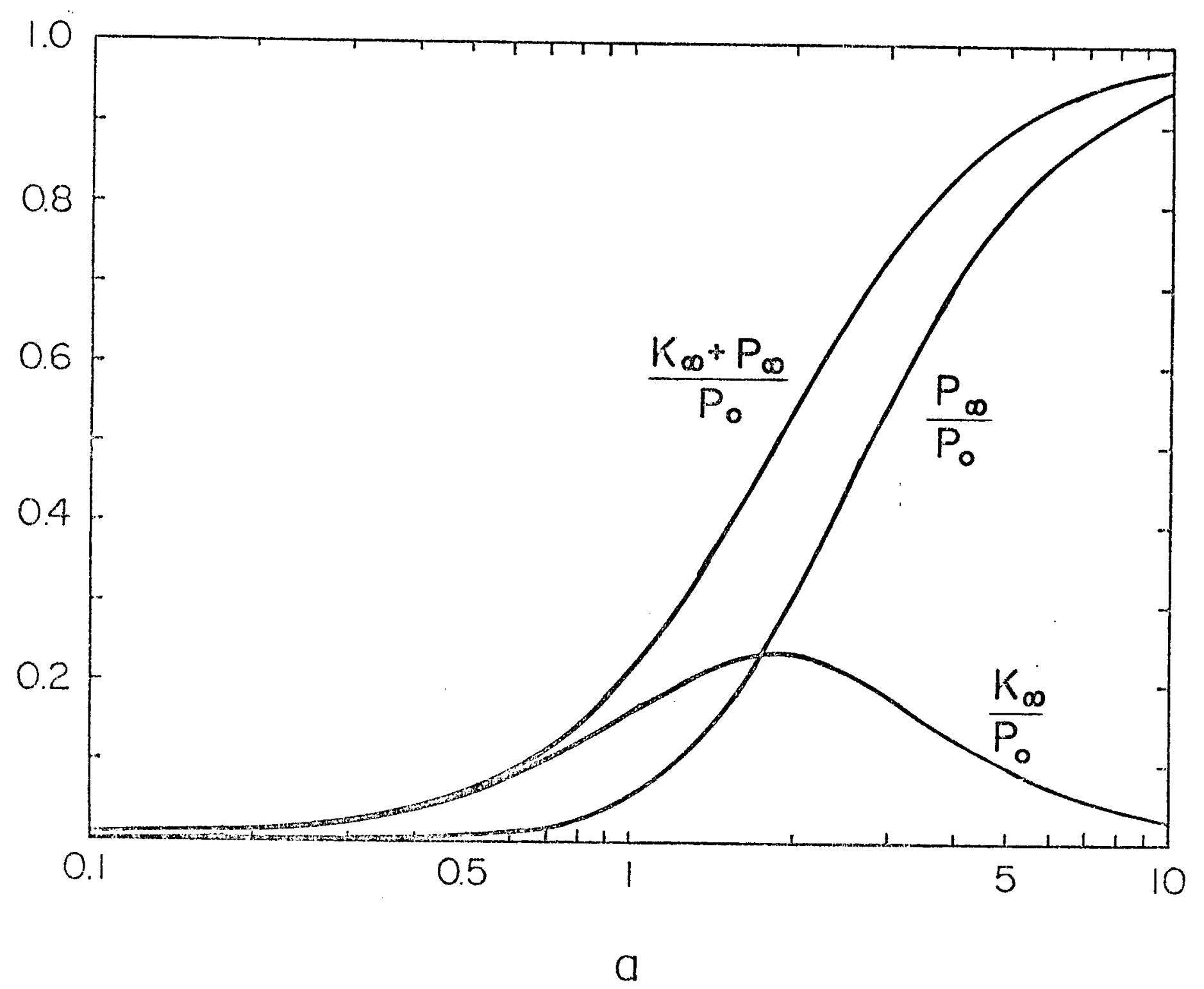

N

Figure 11a. 


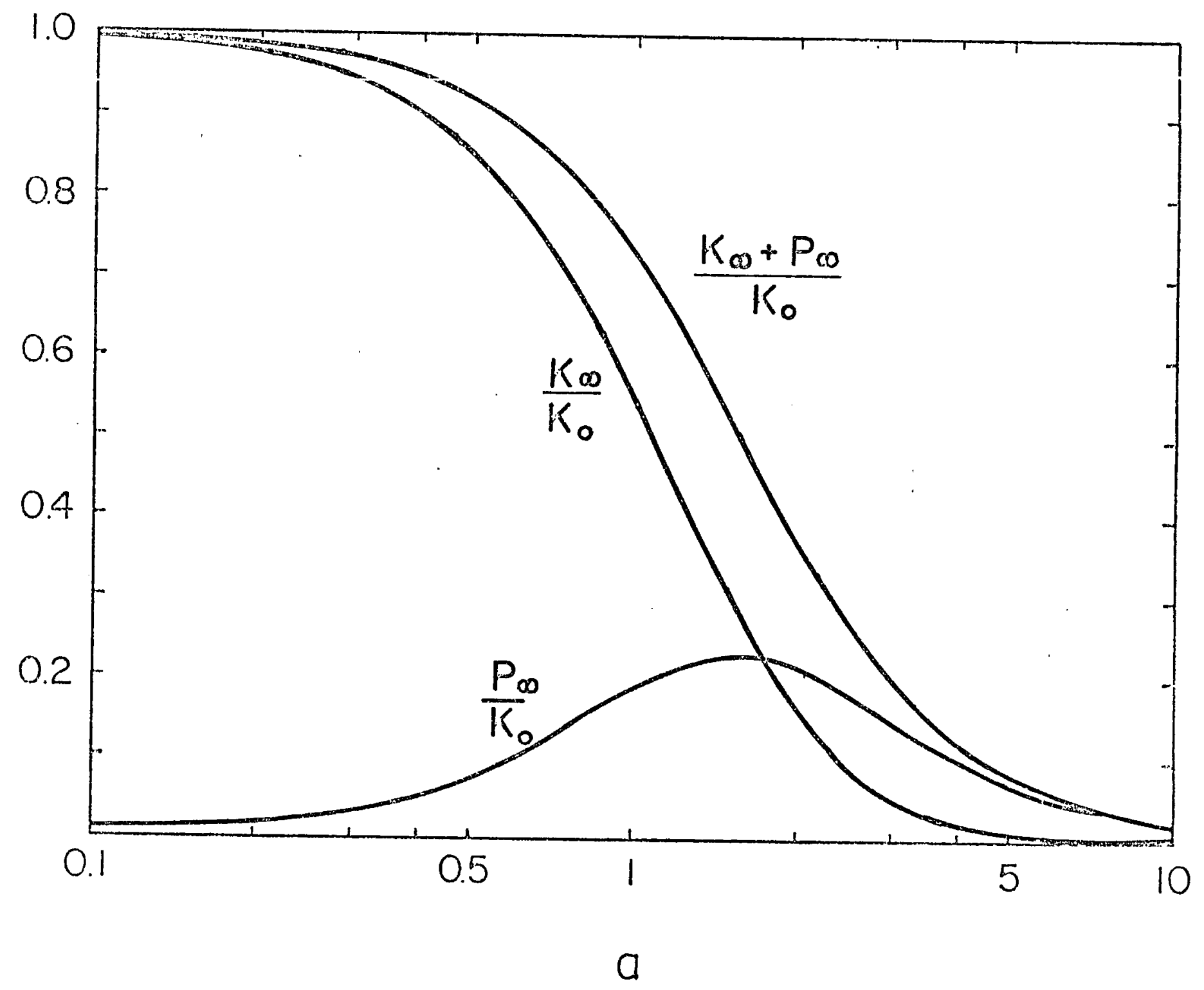

ต

Figure 11b. 


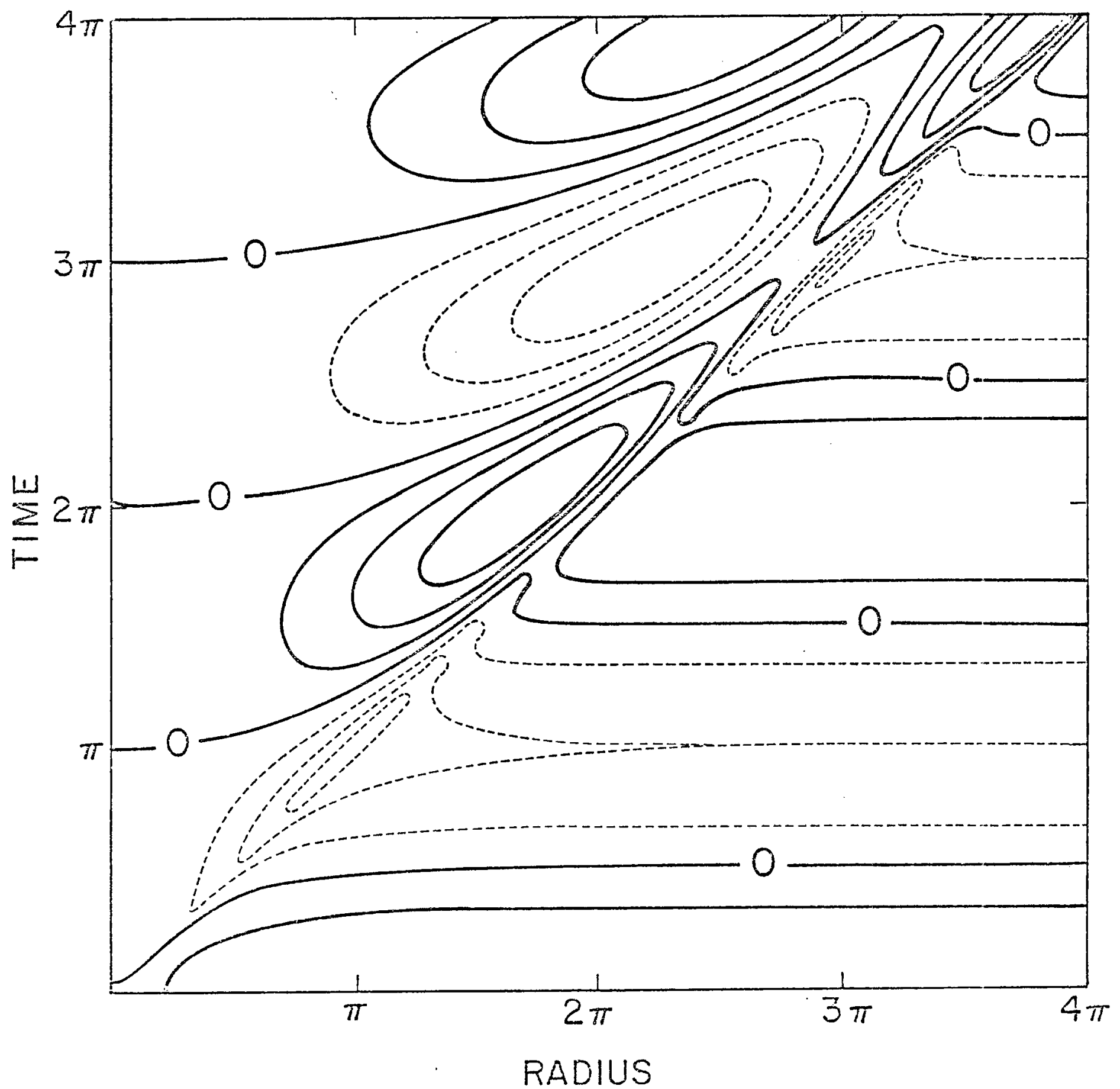

Figure 12. 


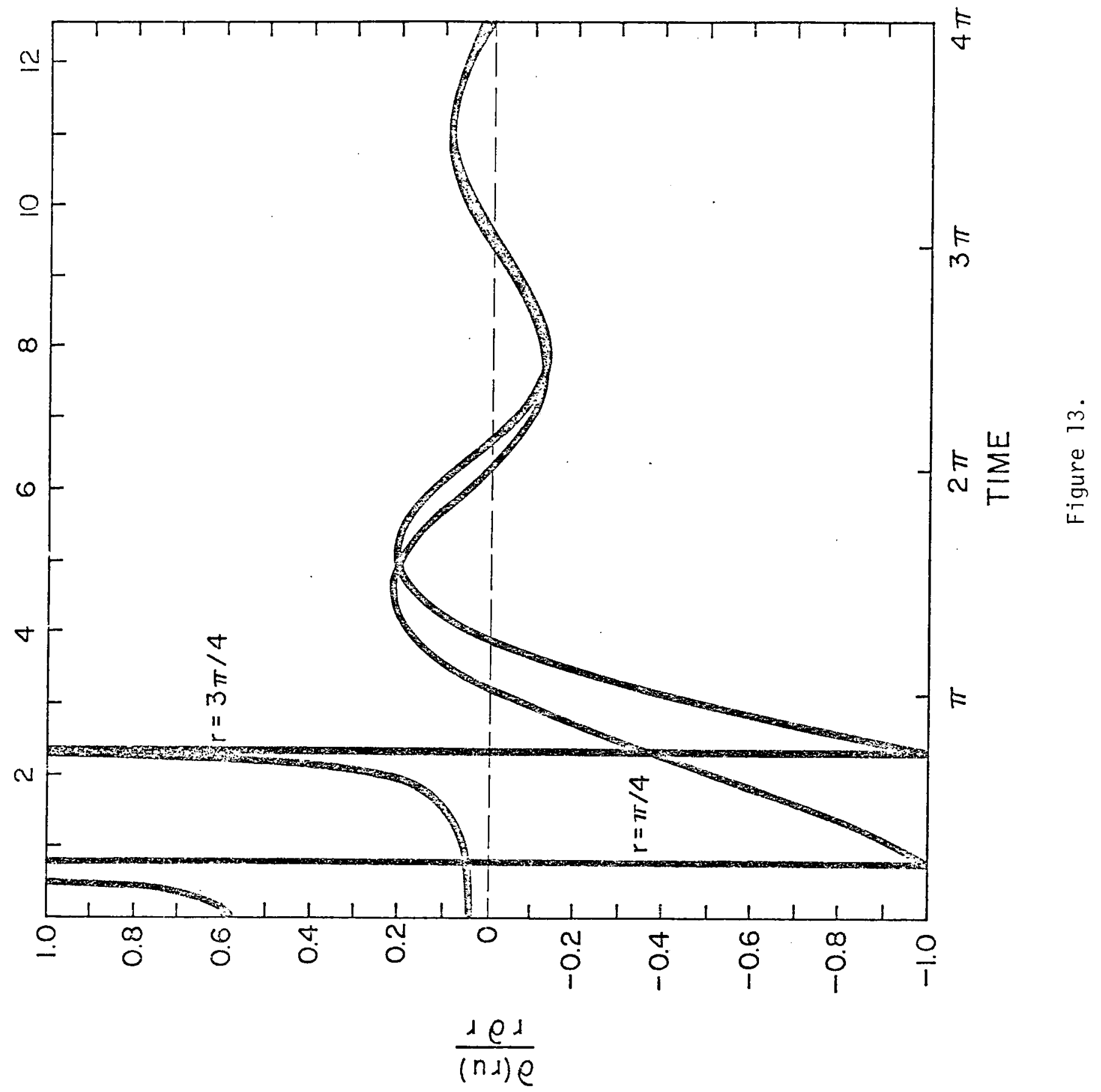




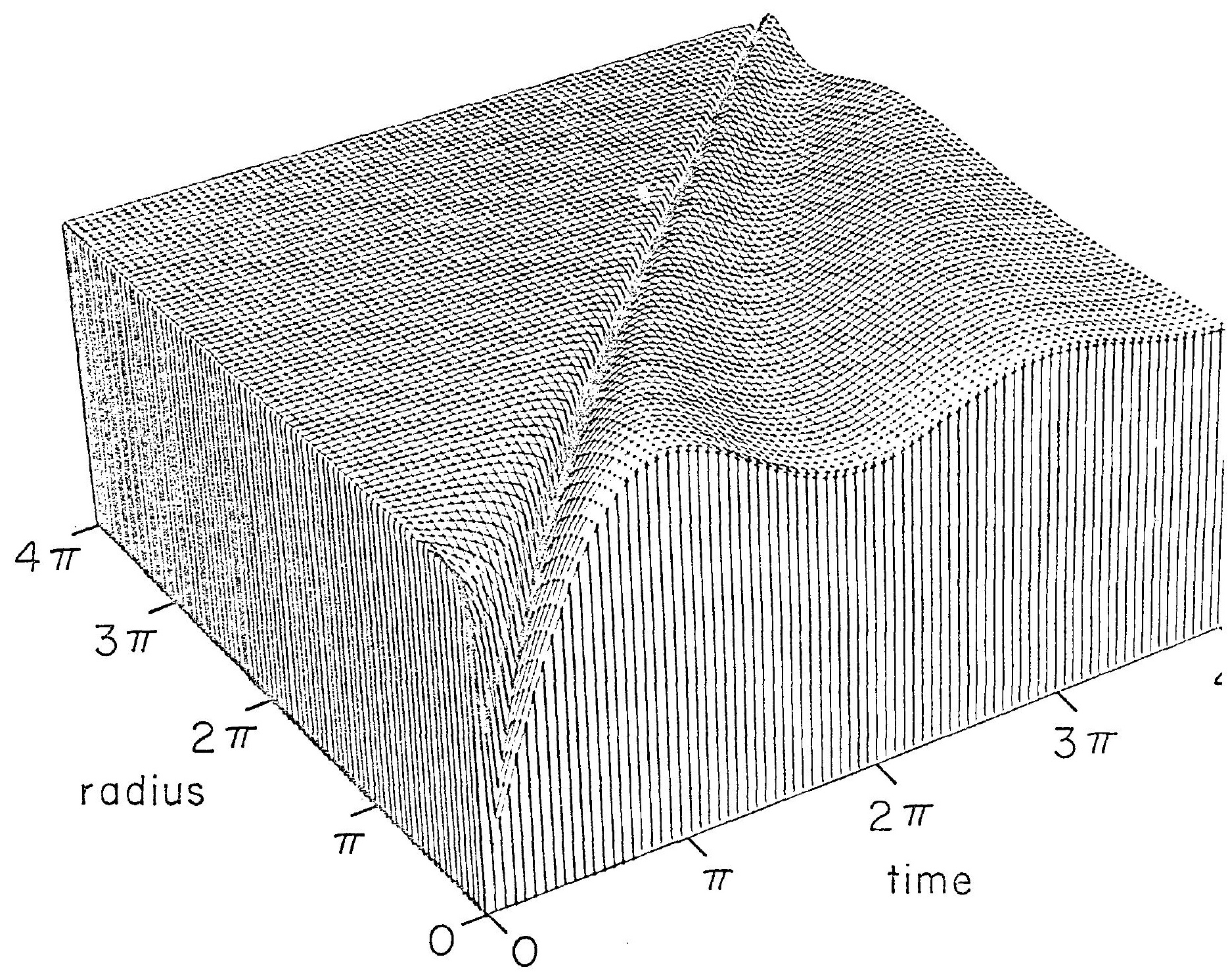

Figure 14. 


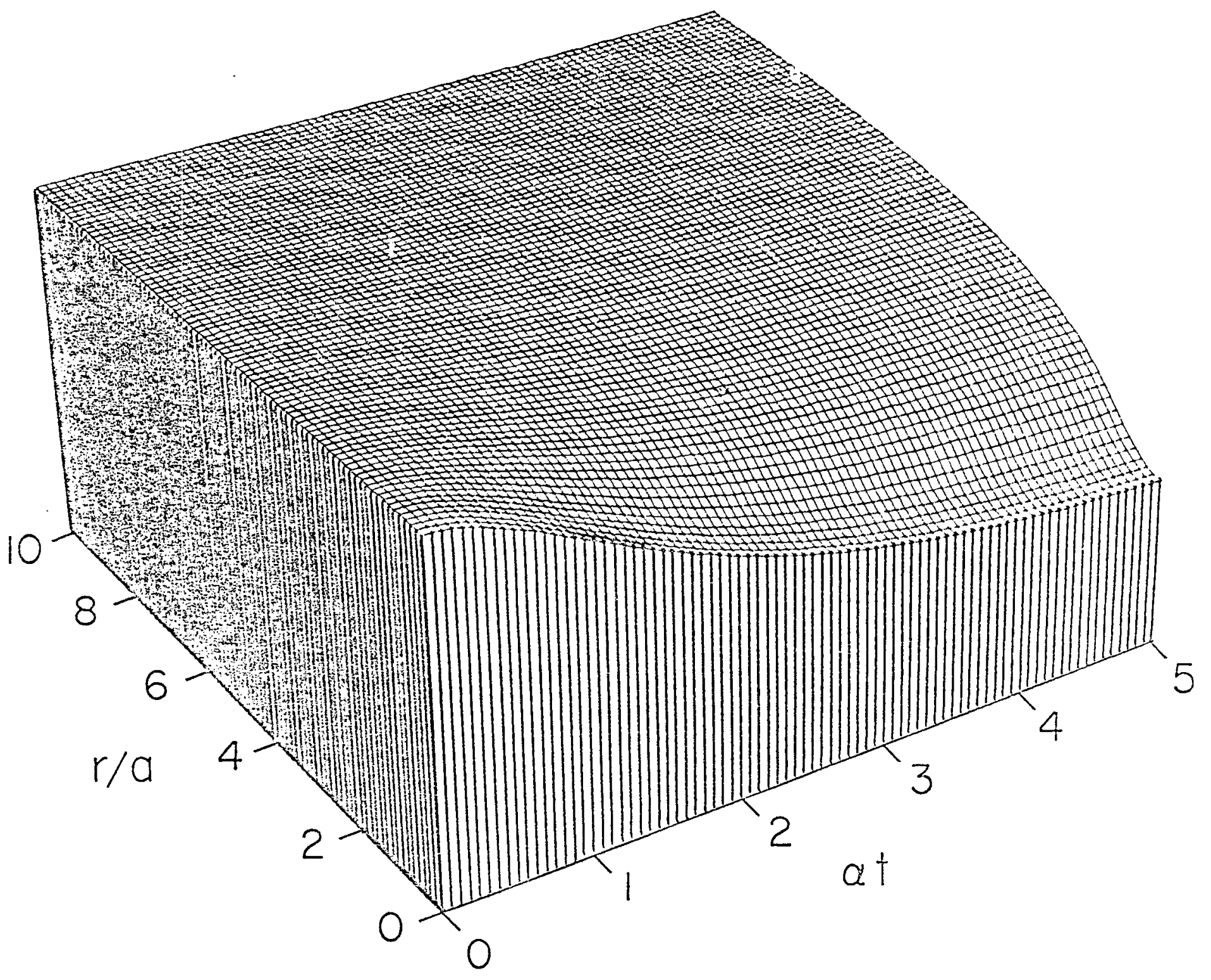

Figure $15 a$. 


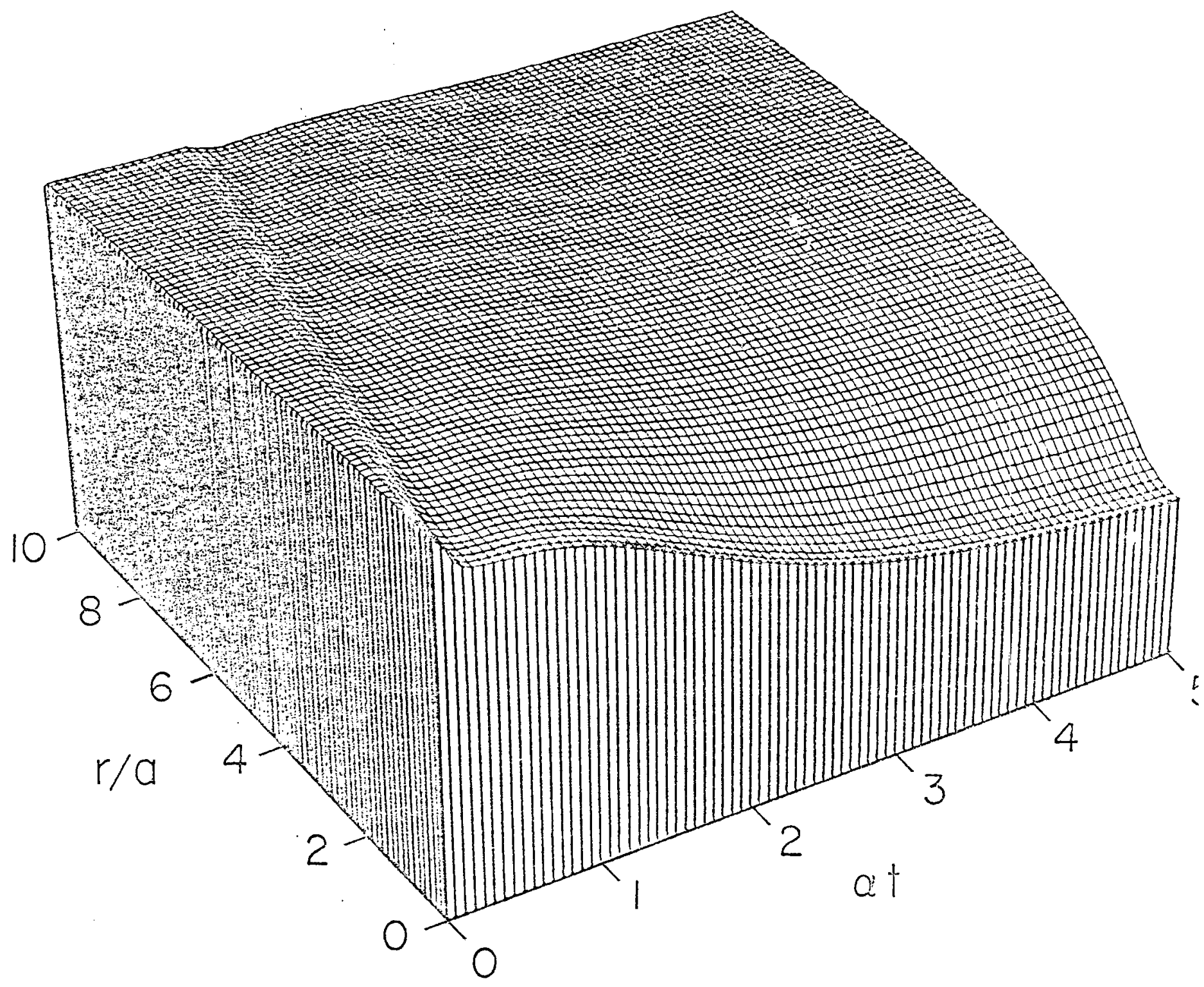

Figure 15b. 


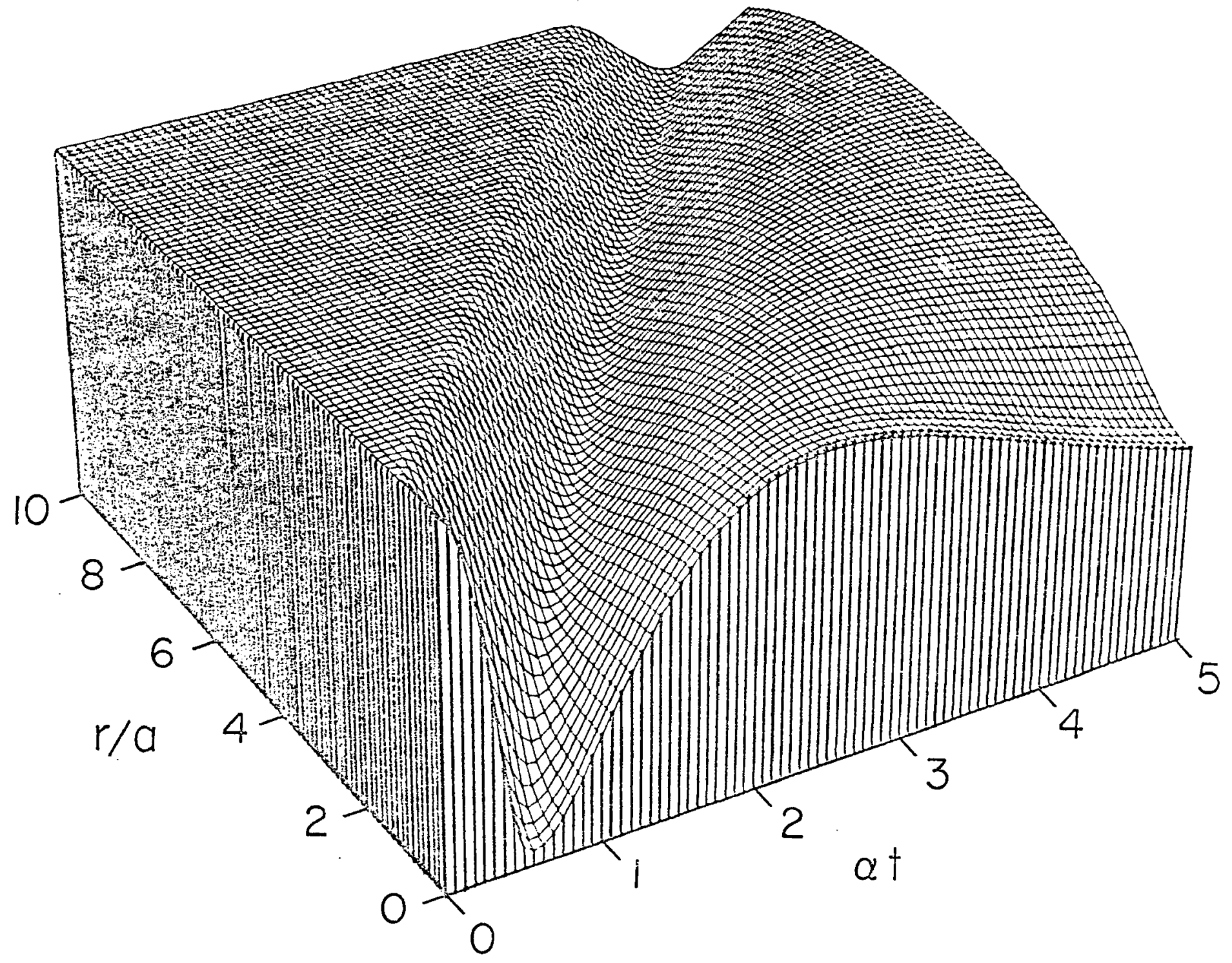

Figure $15 c$. 


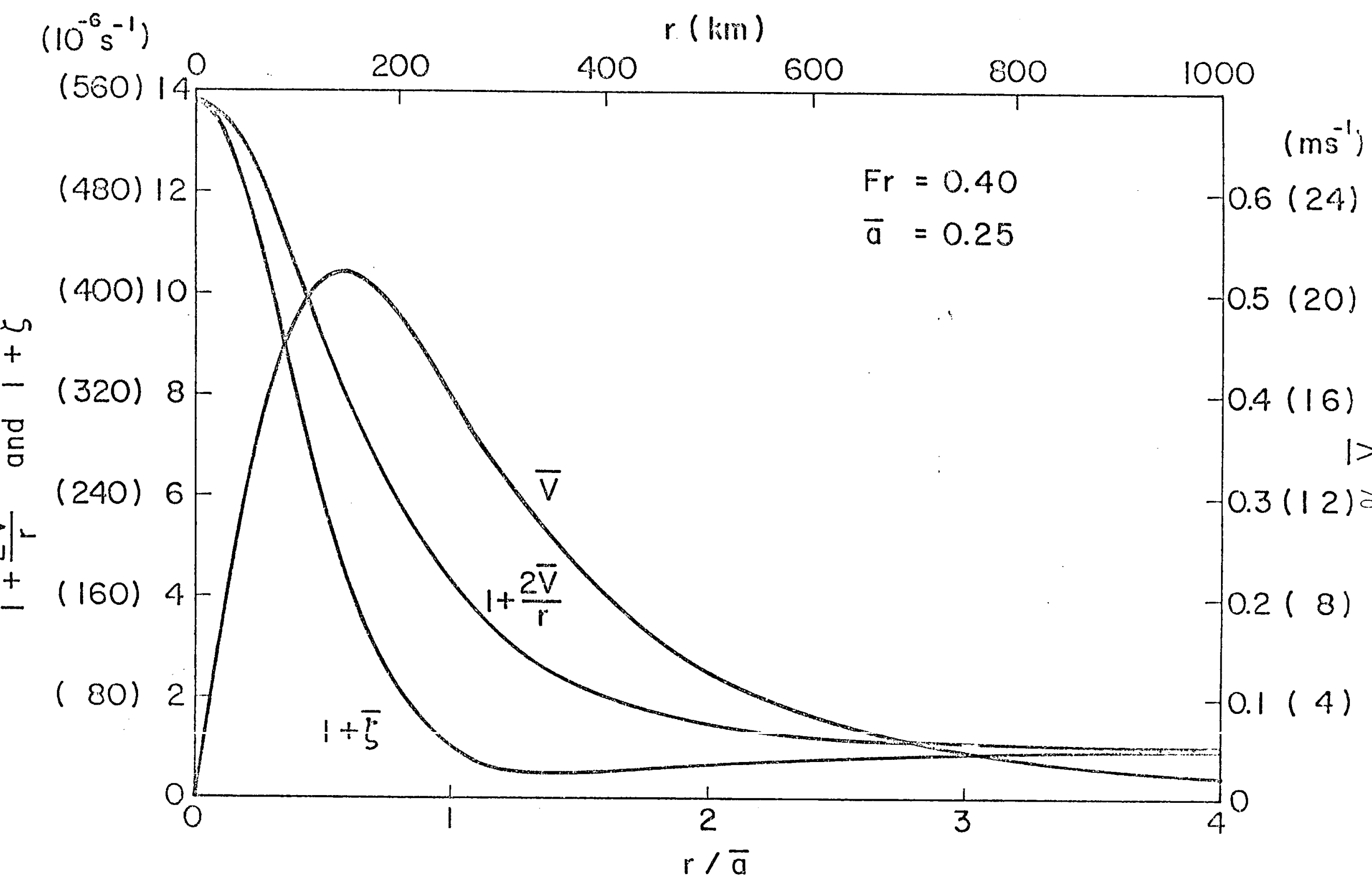

Figure 16. 


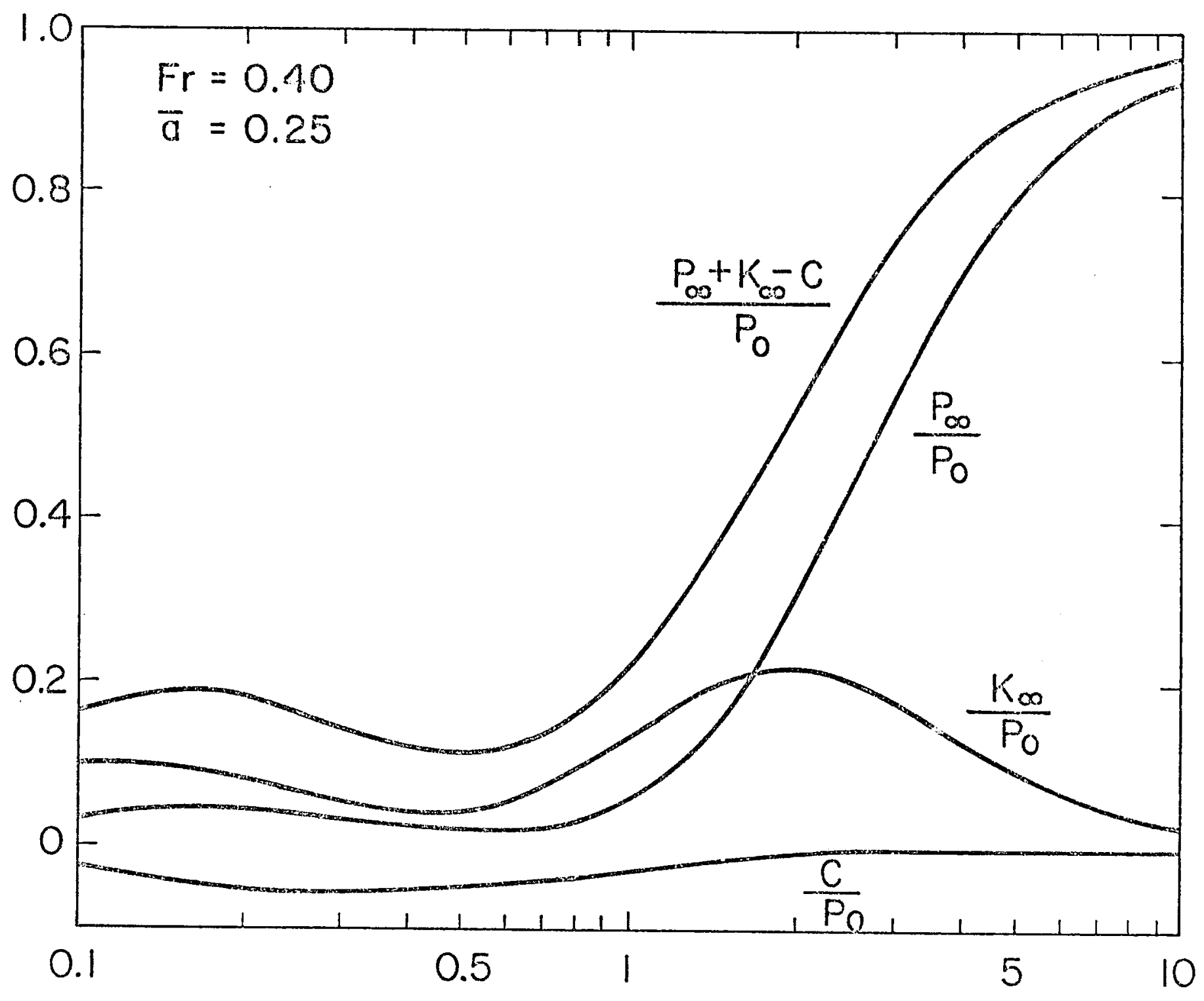

Figure 17a. 


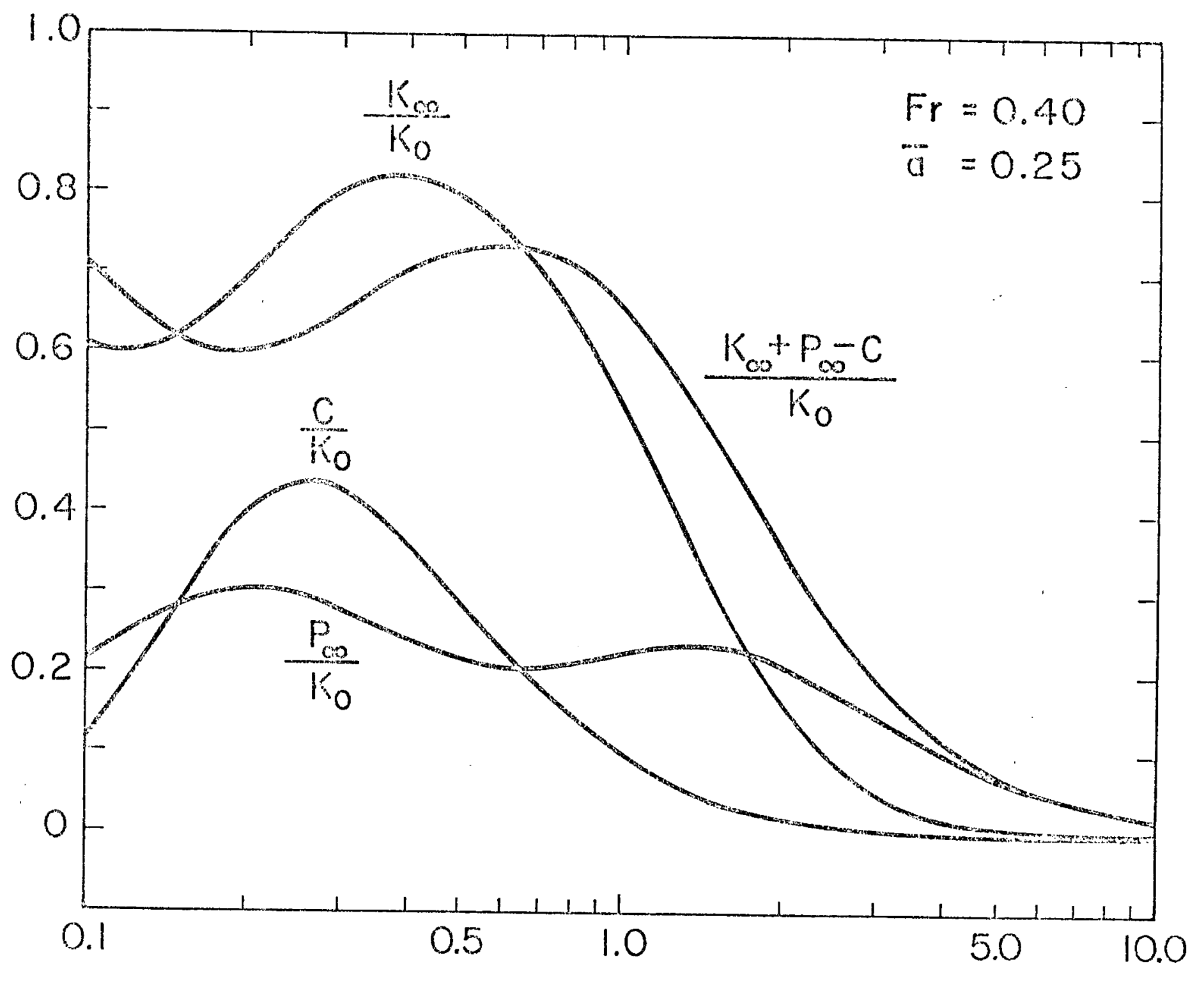

Figure 17b. 


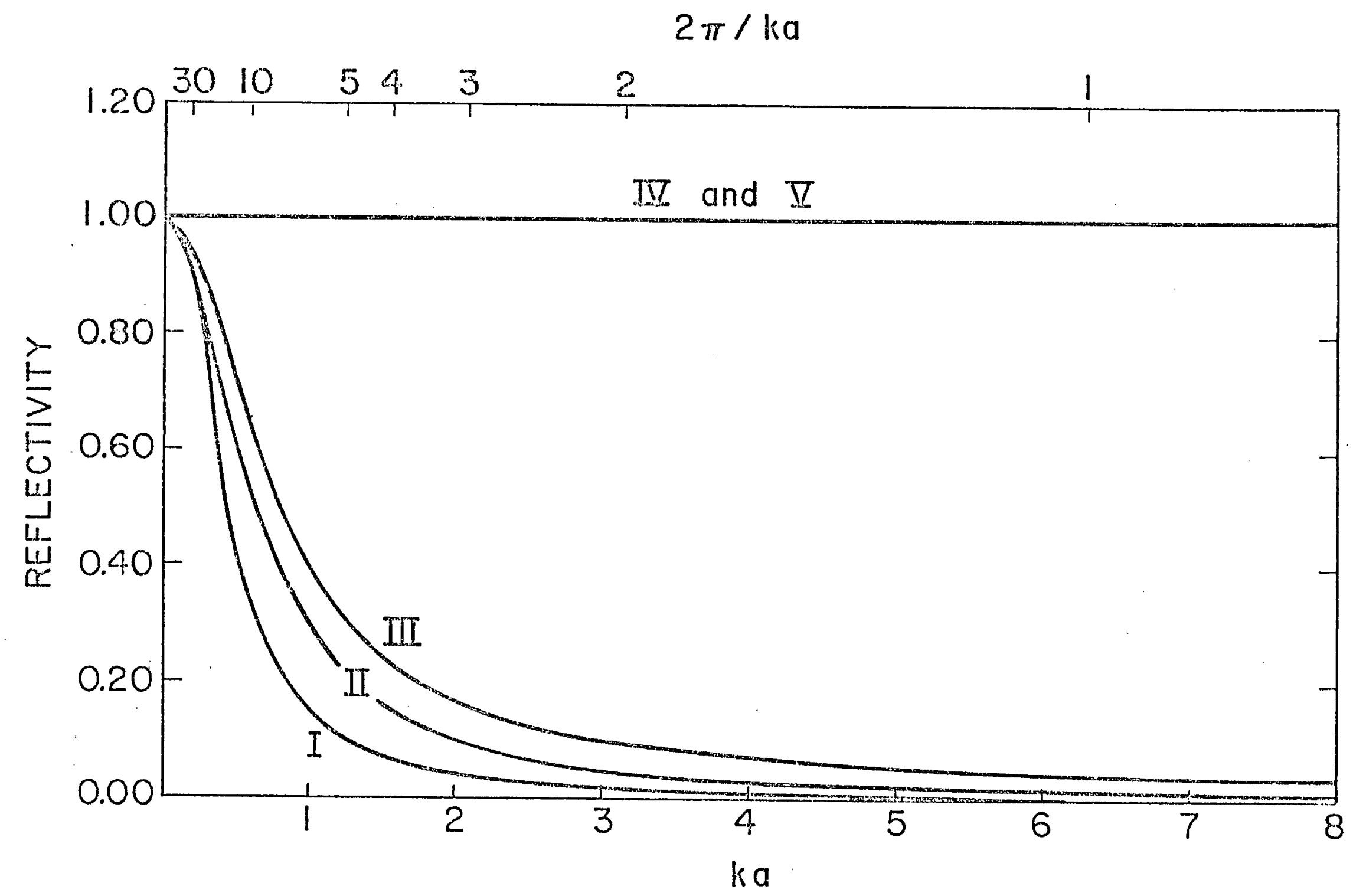

Figure 18. 
BIELIOCRAPHIC OATA

\begin{tabular}{|c|c|}
\hline 4. intle did subitie & $\begin{array}{l}\text { 5. Keport wice } \\
\text { November, } 1979\end{array}$ \\
\hline GEOSTROPHIC ADJUSTMENT IN AN AXISYMMETRIC VORTEX & 6. \\
\hline $\begin{array}{l}\text { 7. Author(s) Wayne H. Schubert, James J. Hack, Pedro L. Silva Dias, } \\
\text { and Scott R. Fulton }\end{array}$ & 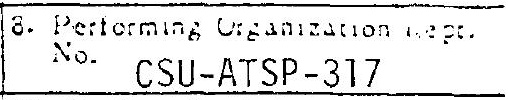 \\
\hline $\begin{array}{l}\text { 9. Pctiorming U: anization kame and dúuess } \\
\text { Department of Atmospheric Science }\end{array}$ & 10. Frojectílasklhuek lnat io. \\
\hline $\begin{array}{l}\text { Colorado State University } \\
\text { Foothills Campus } \\
\text { Fort Collins; C0 } 80523\end{array}$ & $\begin{array}{l}\text { 11. Contraci Giane No. } \\
\text { GARP, GATE Project } \\
\text { Grant No. ATM-7808125 }\end{array}$ \\
\hline 12 Spensoing Uiganization Nime and iujuress & $\begin{array}{l}\text { 13. Thpe of Kepurs \& Fersud } \\
\text { Covered }\end{array}$ \\
\hline National Science Foundation & \\
\hline & 14. \\
\hline
\end{tabular}

15. Supplemenusy Nores

16. Absidic:s

A linearized system of equations for the atmosphere's first internat mode in the vertical is derived. The system governs smal1 amplitude, forced, axisymmetric perturbations on a basic state tangential flow which is independent of height. When the basic flow is at rest, solutions for the transient and final adjusted state are found by the method of Hankel transforms. Two examples are considered, one with an initial top- at potential vorticity and one with an initial Gaussian-type potential vorticity. These two examples, which extend the work of Fischer (1963) and Obukhov (1949), indicate that . he energetical efficiency of cloud cluster scale heating in producing balanced vortex flow is very low, on the order of a few percent. The vast majority of the energy is simp' $y$ partitioned to gravity-inertia waves. In contrast the efficiency of cloud cluster scale vorticity transport is very high.

When the basic state possesses positive relative vorticity in an inner region, the energy partition can be substantially modified, and cloud cluster scale heating can considerably more efficient.

17. Key Hords and Documera trabjis. 17a. Lescripiors

$$
\begin{aligned}
& \text { geostrophic adjustment } \\
& \text { gravity-inertia waves } \\
& \text { tropical cyclones }
\end{aligned}
$$

\begin{tabular}{|c|c|c|c|}
\hline \multirow{2}{*}{$\begin{array}{c}\text { 18. Avaliablity Sratenent } \\
\text {. } \\
\cdots\end{array}$} & \multirow{2}{*}{$\cdot \cdot \cdot$} & 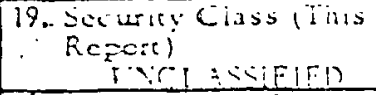 & $\begin{array}{l}\text { 21. No. : Pajes } \\
72\end{array}$ \\
\hline & & 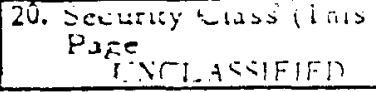 & 22. i'sue \\
\hline
\end{tabular}

17b. Lentiliers/Open-Ended Terms

17c. COStTt Fiels/Group 